\title{
MMC Impedance Modelling and Interaction of Converters in Close Proximity
}

\author{
Yin Chen, Lie Xu, Senior Member, IEEE, Agustí Egea-Àlvarez, Member, IEEE, Benjamin \\ Marshall, Md Rahman, and Oluwole D. Adeuyi
}

\begin{abstract}
This paper develops a small-signal impedance model of modular multilevel converters (MMCs) using harmonic statespace (HSS) method and studies the stability in a multiple converter scenario. In order to simplify analysis on the coupling characteristics between different frequencies in MMCs, the proposed model is developed in the positive-negative-zero (PNO) sequence-frame, where the zero-sequence current in three-phase three-wire system is directly set to zero without introducing complicated method. A simple 2 by 2 admittance matrix in PNOframe is extracted from the MMC small-signal model for ease of system stability analysis. Using the developed impedance model, the multi-infeed interaction factor (MIIF) measure is adopted to analyze the most significant interactions for multi-infeed converter systems to be prioritized. Different outer-loop controllers are adopted and compared in the analysis to illustrate the effect of different control modes on converter impedance and system stability. Analytical studies and time-domain simulation results are provided to validate the proposed model and stability analysis.
\end{abstract}

Index Terms- Admittance, harmonic state-space (HSS), modular multilevel converter (MMC), stability.

\section{INTRODUCTION}

With rapidly increased penetration of renewable energy and distributed generation, and the increased use of HVDC systems for interconnection and renewable integration, system stability of grid-connected converters becomes a significant challenge [1]. Hence, effective methods to identify the source of resonance and to mitigate the stability problems becomes critically important. To assess the system stability and dynamic interactions between grid and converters, the impedance-based stability analysis [2][3] is an effective method of identifying potential frequencies of disturbance to which an individual converter may be vulnerable to destabilizing behavior.

Therefore, an accurate impedance modelling of converters is required. Modular multilevel converter (MMC) is now being widely used for HVDC systems [4-6]. However, due to its inherently complex behavior such as internal circulating current and submodule (SM) capacitor voltage ripple, accurately modelling the impedance or admittance of MMC is a challenging task [7][8]. The consequences of not modelling

This work is supported in part by the UK National HVDC Center. Y. Chen, L. Xu, and A. Egea are with the Department of Electronic and Electrical Engineering, University of Strathclyde, Glasgow G1 1XW, U.K. (e-mail: yin.chen@strath.ac.uk, lie.xu@strath.ac.uk, agusti.egea@strath.ac.uk ).

B. Marshall, Md Rahman, O. D. Adeuyi are with the UK National HVDC Centre, Cumbernauld, G68 0FQ, UK. (email: Benjamin.Marshall@sse.com,

Oluwole.Adeuyi@sse.com ) these aspects could lead to a displacement or deletion of frequencies of interaction and a mis-estimation of the magnitude of the impedance changes at those points.

Various studies have been carried out on developing MMC impedance models. In [9], an analytical sequence impedance model of a three-phase MMC is derived with the internal MMC dynamics, following the same approach used for 2-level VSCs. However, the $2^{\text {nd }}$ harmonic in the arm current and PLL are not considered in the model. In [10], the AC side input admittances of the MMC under various control strategies are derived, though the circulating current controller is not included. Reference [11] focuses on the impact of different currentcontrol schemes on the shape of MMC admittance, considering the $2^{\text {nd }}$ internal harmonic current. However, a large resistive load is added at the AC side to provide increased passive damping, so that high-order harmonics are not presented in the system and not considered in [11]. However, in real systems, such strong passive damping does not exist and harmonic or inter-harmonic resonances are a major concern and must be modelled.

The Harmonic State-Space (HSS) method proposed to analyze linear time-periodic (LTP) system [12], models not only the steady-state harmonics in LTP systems, but also the dynamics of the harmonics during transients. Consequently, HSS method has been widely used to model power networks and converters, e.g., static synchronous compensators [13], LCC converters [12], transmission lines [14], and two-level VSCs [15]. Recently, HSS method has been used to model MMC impedance considering the impact of the internal harmonics [16]-[18]. Since the Fourier coefficients matrices in the HSS model are diagonal-constant matrices (Toeplitz matrices) [16], the MMC small-signal model based on HSS can be easily extended to any harmonic order. Hence, the dynamics of high-order harmonics in MMC can be fully considered.

However, various problems and limitations still exist in the proposed HSS-based MMC small-signal modelling methods [16]-[18]. In [16], a single-phase MMC model is developed and the impedance that reflects the voltage and current at the same frequency is derived but the couplings at different frequencies generated by the internal harmonics of MMC, are not taken into account. Impedance models of three-phase four-wire MMC systems, in which both the MMC DC mid-point and the AC neutral point are grounded providing a circulation path for the zero-sequence current, are derived in [17][18]. However, in reality, MMC systems are likely to be configured as an equivalent three-phase three-wire system without the low impedance path for the zero-sequence current. To describe the MMC zero-sequence current on the MMC AC side in threephase three-wire systems, zero-sequence voltage compensation is proposed to add into the single-phase model in [17] and the 
single-phase impedance is obtained for three-phase system stability assessment. The MMC controllers in the models also adopt the proportional resonant (PR) controller in the abcframe, implying that the controls for phase $\mathrm{a}, \mathrm{b}$, and $\mathrm{c}$ are totally independent and identical. However, for MMC controller implemented in $\alpha \beta$-frame or $\mathrm{dq}$-frame, the single-phase modelling method is inadequate and is thus unsuitable for threephase MMC system.

In the latest study on MMC impedance modelling, reference [19] provides a comprehensive three-phase HSS model of MMC. Similar to [17], the DC mid-point voltage is compensated using the AC neutral point voltage to eliminate the zero-sequence current. However, when developing the small-signal model, the expression of the neutral point voltage involves the steady-state value and the perturbation variables of three-phase voltages as well as the control signal of the arms, which lead to an extra complex calculation in the HSS model. In [19], the complex vector representation of the controllers in dq-frame obtained based on the transfer function, has to be transformed to the $\alpha \beta$-frame before being integrated into the MMC model to obtain the impedance in the positive-negativezero (PN0) sequence frame, thus leading to complicated transformation and calculation.

In addition, the MMC impedance obtained in [19] is a 10 by 10 matrix. In order to simplify the process of stability assessment, a single input and single output (SISO) equivalent impedance of the MMC is derived by considering the grid side impedance. However, if the grid structure is more complex, e.g., there are other converters connected to the grid in close proximity, the grid impedance seen by the MMC will also become complicated. Thus it is difficult to simplify the 10 by 10 matrix of MMC impedance to a SISO equivalent. Therefore, a MMC impedance independent of the grid side impedance and in simple form is more beneficial for system stability assessment with multiple converters. MMC represented by 2 by 2 impedance matrix in modified sequence-domain [20] is developed in [21][22]. However, in the modified sequencedomain the frequencies of the coupling admittance cannot be represented. Moreover, unlike the impedance in the sequence domain, the MMC impedance in the modified sequence domain cannot be measured directly in time domain due to the existence of frequency shift between the modified sequence domain and sequence domain. In [23], a MMC 2 by 2 impedance matrix is derived to capture the characteristics of frequency coupling in sequence frame. However, the work focuses on the coupling between the AC system and DC system of the MMC and the dynamic of the PLL in AC side is not considered.

Considering the limitations in the existing modelling approach and limited work on assessing system stability with multiple MMCs, the main contributions of this paper are:

- Addressing the non-existence zero sequence current in three-phase three-wire systems, instead of adding a compensation voltage [17][19], the zero-sequence current is directly forced to zero thus providing a simplified modelling approach;

- When mapping the dq-controllers, their transfer functions are directly transformed from dq to the $\mathrm{PN} 0$-frame which significantly simplifies the modelling process compared to [19];

- Simplification of the high-dimensional MMC admittance to a 2 by 2 matrix based on the characteristics of MMC harmonics in PNO-frame. The MMC impedance independent of the grid side impedance with simpler form can be more suitable for system stability assessment especially when multiple converters are considered;

- Interaction of converters in close proximity is studied using the developed models and system stability assessment in case of multiple MMCs in a network are carried out considering the multi-infeed interaction factor (MIIF).

The rest of the paper is organized as follows. Section II presents the HSS modelling procedure of three-phase MMC. In Section III, the small-signal admittance of MMC in PNO-frame is derived while Section IV verifies the proposed model and conducts the stability assessment. Section V analyzes the stability of converters in close proximity and Section VI draws the conclusions.

\section{HSS MODELLING OF THREE-PHASE MMC}

\section{A. Linearizing MMC model in abc-frame}

The equivalent circuit of the MMC average model [24][25] is depicted in Fig. 1, where a lumped capacitor $C_{m}$ and a voltage source are used to mimic the dynamic of each arm. Take phase $a$ as an example, $V_{a}$ and $Z_{g}$ represent the grid voltage and impedance seen at the MMC AC terminals, i.e., the impedance of converter transformer is incorporated into $Z_{g} . \Delta v_{p a}$ is a small AC perturbation voltage. $v_{\text {cua }}^{\Sigma}$ and $v_{\text {cla }}^{\Sigma}$ are the sum of the capacitor voltages of the SMs in the upper and lower arms, respectively. The upper and lower arm current are $i_{u a}$ and $i_{l a}$, the arm voltage $v_{u a}$ and $v_{l a}$, and modulation control signal $n_{u a}$ and $n_{l a} . v_{g a}$ and $i_{g a}$ are the voltage and current on the AC side of the MMC, respectively. The DC voltage $V_{d c}$ is assumed to be constant. Since the system is in a three-phase three-wire connection, the voltage of the DC mid-point is $v_{n}$.

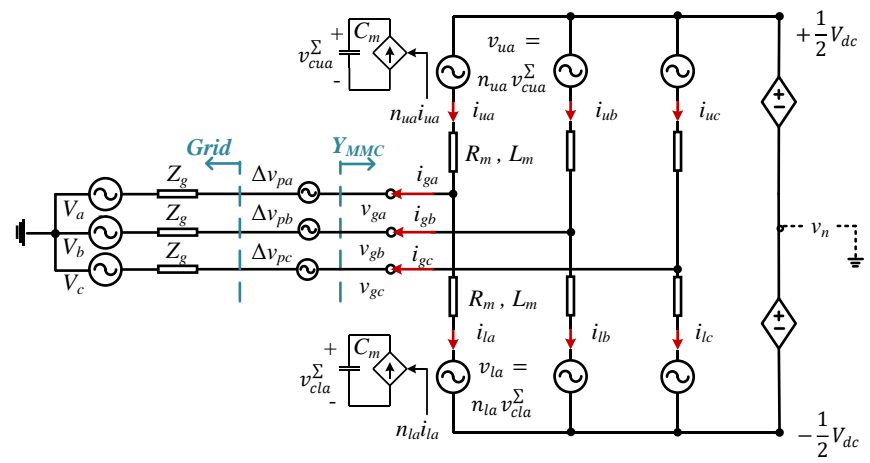

Fig. 1 Equivalent circuit of a three-phase MMC

For ease of analysis, the three-phase quantities are defined in 3 by 1 matrices as $\mathbf{v}_{g a b c}, \mathbf{i}_{g a b c}, \mathbf{v}_{c u a b c}^{\sum}, \mathbf{v}_{c l a b c}^{\Sigma}, \mathbf{v}_{u a b c}, \mathbf{i}_{u a b c}, \mathbf{v}_{l a b c}, \mathbf{i}_{l a b c}$, $\mathbf{i}_{c a b c}, \mathbf{V}_{d c}$, and $\mathbf{v}_{n}$, whereas $\mathbf{n}_{u a b c}$ and $\mathbf{n}_{l a b c}$ are 3 by 3 diagonal matrices.

For a three-phase MMC, the relationship between the arm voltage and the equivalent capacitor voltage of the SMs can be expressed as:

$$
\mathbf{v}_{\text {uabc }}=\mathbf{n}_{\text {uabc }} \cdot \mathbf{v}_{\text {cuabc }}^{\Sigma}, \quad \mathbf{v}_{\text {labc }}=\mathbf{n}_{\text {labc }} \cdot \mathbf{v}_{\text {clabc }}^{\Sigma}
$$

The internal dynamics between equivalent capacitor voltage of SMs and the arm current are depicted as: 
This paper is a post-print of a paper submitted to and accepted for publication in IEEE Journal of Emerging and Selected Topics in Power Electronics and is subject to Institution of Electrical and Electronic Engineering Copyright. The copy of record is available at IEEE Xplore Digital Library.

$$
C_{m} \frac{d \mathbf{v}_{\text {cuabc }}^{\Sigma}}{d t}=\mathbf{n}_{\text {uabc }} \cdot \mathbf{i}_{\text {uabc }}, \quad C_{m} \frac{d \mathbf{v}_{\text {clabc }}^{\Sigma}}{d t}=\mathbf{n}_{\text {labc }} \cdot \mathbf{i}_{\text {labc }}
$$

The arm voltages $\mathbf{v}_{\text {uabc }}$ and $\mathbf{v}_{\text {labc }}$, and the capacitor voltages $\mathbf{v}_{\text {cuabc }}^{\Sigma}$ and $\mathbf{v}_{\text {clabc }}^{\Sigma}$ all contains multiple harmonics [24]. It indicates that MMC has multi-frequency responses due to its significant steady-state harmonic components in the arm currents and capacitor voltages.

The common-mode current that circulates inside the arms and the $\mathrm{AC}$ side current are denoted as:

$$
\begin{gathered}
\mathbf{i}_{\text {cabc }}=\left(\mathbf{i}_{\text {uabc }}+\mathbf{i}_{\text {labc }}\right) / 2 \\
\mathbf{i}_{g a b c}=\mathbf{i}_{\text {uabc }}-\mathbf{i}_{\text {labc }}
\end{gathered}
$$

The voltage on the AC terminal of MMC and the currents and voltages of the arms have the following relationship:

$$
\left\{\begin{array}{l}
\mathbf{v}_{g a b c}+L_{m} \frac{d \mathbf{i}_{\text {uabc }}}{d t}+R_{m} \mathbf{i}_{\text {uabc }}+\mathbf{v}_{\text {uabc }}=\frac{\mathbf{V}_{d c}}{2}+\mathbf{v}_{n} \\
\mathbf{v}_{g a b c}-L_{m} \frac{d \mathbf{i}_{\text {labc }}}{d t}-R_{m} \mathbf{i}_{\text {labc }}-\mathbf{v}_{\text {labc }}=-\frac{\mathbf{V}_{d c}}{2}+\mathbf{v}_{n}
\end{array}\right.
$$

where $\mathbf{v}_{n}=\left[v_{n}, v_{n}, v_{n}\right]^{\mathrm{T}}$ and the DC mid-point voltage $v_{n}$ is obtained as [17]

$$
v_{n}=\frac{n_{u a} v_{c u a}^{\Sigma}-n_{l a} v_{c l a}^{\Sigma}+n_{u b} v_{c u b}^{\Sigma}-n_{l a} v_{c l b}^{\Sigma}+n_{u c} v_{c u c}^{\Sigma}-n_{l c} v_{c l c}^{\Sigma}}{6}
$$

Combining (1)-(4) with (5) and considering small perturbations, the MMC small-signal state-space model in abcframe can be expressed as:

$$
\begin{aligned}
& \int \frac{d \Delta \mathbf{i}_{\text {cabc }}}{d t}=-\frac{R_{m}}{L_{m}} \Delta \mathbf{i}_{\text {cabc }}-\frac{\mathbf{N}_{\text {uabc }}}{2 L_{m}} \Delta \mathbf{V}_{\text {cuabc }}^{\sum}-\frac{\mathbf{N}_{\text {labc }}}{2 L_{m}} \Delta \mathbf{V}_{\text {clabc }}^{\sum}-\frac{\mathbf{V}_{\text {cuabc }}^{\Sigma}}{2 L_{m}} \Delta \mathbf{n}_{\text {uabc }} \\
& -\frac{\mathbf{V}_{\text {clabc }}^{\Sigma}}{2 L_{m}} \Delta \mathbf{n}_{\text {labc }} \\
& \frac{d_{\Delta} \mathbf{i}_{\text {gabc }}}{d t}=-\frac{R_{m}}{L_{m}} \Delta \mathbf{i}_{g a b c}-\frac{\mathbf{N}_{\text {uabc }}}{L_{m}} \Delta \mathbf{V}_{\text {cuabc }}^{\Sigma}+\frac{\mathbf{N}_{\text {labc }}}{L_{m}} \Delta \mathbf{V}_{\text {clabc }}^{\Sigma}-\frac{\mathbf{V}_{\text {cuabc }}^{\Sigma}}{L_{m}} \Delta \mathbf{n}_{\text {uabc }} \\
& +\frac{\mathbf{V}_{c l a b c}^{\Sigma}}{L_{m}} \Delta \mathbf{n}_{\text {labc }}-\frac{2}{L_{m}} \Delta \mathbf{v}_{p a b c}+\frac{2}{L_{m}} \Delta \mathbf{v}_{n} \\
& \frac{d \Delta \mathbf{v}_{\text {cuabc }}^{\Sigma}}{d t}=\frac{\mathbf{N}_{\text {uabc }}}{C_{m}} \Delta \mathbf{i}_{c a b c}+\frac{\mathbf{N}_{u a b c}}{2 C_{m}} \Delta \mathbf{i}_{g a b c}+\frac{\mathbf{I}_{c a b c}}{C_{m}} \Delta \mathbf{n}_{u a b c}+\frac{\mathbf{I}_{g a b c}}{2 C_{m}} \Delta \mathbf{n}_{u a b c} \\
& \frac{d \Delta \mathbf{v}_{c l a b c}^{\Sigma}}{d t}=\frac{\mathbf{N}_{l a b c}}{C_{m}} \Delta \mathbf{i}_{c a b c}-\frac{\mathbf{N}_{l a b c}}{2 C_{m}} \Delta \mathbf{i}_{\text {gabc }}+\frac{\mathbf{I}_{c a b c}}{C_{m}} \Delta \mathbf{n}_{\text {labc }}-\frac{\mathbf{I}_{g a b c}}{2 C_{m}} \Delta \mathbf{n}_{\text {labc }}
\end{aligned}
$$

where variables in capital form denote the values at the steadystate operation point and variables with $\Delta$ denote the small perturbations. The matrix $\Delta \mathbf{v}_{n}$ is equal to $\left[\Delta v_{n}, \Delta v_{n}, \Delta v_{n}\right]^{\mathrm{T}}$ and the component $\Delta v_{n}$ can be derived by linearizing (6) as:

$$
\begin{aligned}
\Delta v_{n}= & \frac{N_{u a} \Delta v_{c u a}^{\Sigma}-N_{l a} \Delta v_{c l a}^{\Sigma}+\Delta n_{u a} V_{c u a}^{\Sigma}-\Delta n_{l a} V_{c l a}^{\Sigma}}{6} \\
& +\frac{N_{u b} \Delta v_{c u b}^{\Sigma}-N_{l a} \Delta v_{c l b}^{\Sigma}+\Delta n_{u b} V_{c u b}^{\Sigma}-\Delta n_{l a} V_{c l b}^{\Sigma}}{6} \\
& +\frac{N_{u c} \Delta v_{c u c}^{\Sigma}-N_{l c} \Delta v_{c l c}^{\Sigma}+\Delta n_{u c} V_{c u c}^{\Sigma}-\Delta n_{l c} V_{c l c}^{\Sigma}}{6}
\end{aligned}
$$

\section{B. Transforming small-signal model from abc- to PNO-frame}

The PNO-frame has been chosen to study the system stability since in this frame different harmonics can be effectively tracked for a three-phase system [26]. The transformation matrix $\mathbf{P}$ is adopted to realize the transformation from abc- to PNO-frame [12] as:

$$
\mathbf{P}=\left[\begin{array}{ccc}
1 & e^{j 2 \pi / 3} & e^{j 4 \pi / 3} \\
1 & e^{j 4 \pi / 3} & e^{j 2 \pi / 3} \\
1 & 1 & 1
\end{array}\right]
$$

Applying (9), (7) can be rewritten in PN0-frame as:

$$
\begin{aligned}
& \int \frac{d \Delta \mathbf{i}_{c P N 0}}{d t}=-\frac{R_{m}}{L_{m}} \Delta \mathbf{i}_{c P N 0}-\frac{\mathbf{P N}_{u a b b} \mathbf{P}^{-1}}{2 L_{m}} \Delta \mathbf{v}_{c u P N 0}^{\Sigma}-\frac{\mathbf{P N}_{l a b c} \mathbf{P}^{-1}}{2 L_{m}} \Delta \mathbf{v}_{c l P N 0}^{\Sigma} \\
& -\frac{\mathbf{P} \mathbf{V}_{\text {cuabc }}^{\sum} \mathbf{P}^{-1}}{2 L_{m}} \Delta \mathbf{n}_{u P N 0}-\frac{\mathbf{P} \mathbf{V}_{\text {clabc }}^{\sum} \mathbf{P}^{-1}}{2 L_{m}}{ }{ }_{l P N 0} \\
& \frac{d \Delta \mathbf{i}_{g P N 0}}{d t}=-\frac{R_{m}}{L_{m}} \mathbf{C}_{z} \Delta \mathbf{i}_{g P N 0}-\frac{\mathbf{P} \mathbf{N}_{u a b c} \mathbf{P}^{-1}}{L_{m}} \Delta \mathbf{v}_{c u P N 0}^{\Sigma}+\frac{\mathbf{P} \mathbf{N}_{l a b c} \mathbf{P}^{-1}}{L_{m}} \Delta \mathbf{v}_{c l P N 0}^{\Sigma} \\
& -\frac{\mathbf{P V}_{\text {cubbc }}^{\Sigma} \mathbf{P}^{-1}}{L_{m}} \Delta \mathbf{n}_{u P N 0}+\frac{\mathbf{P V}_{\text {clabc }}^{\Sigma} \mathbf{P}^{-1}}{L_{m}} \Delta \mathbf{n}_{l P N 0}-\frac{2}{L_{m}} \Delta \mathbf{v}_{p P N 0} \\
& \frac{d \Delta \mathbf{v}_{c u P N 0}^{\Sigma}}{d t}=\frac{\mathbf{P N}_{u a b c} \mathbf{P}^{-1}}{C_{m}} \Delta \mathbf{i}_{c P N 0}+\frac{\mathbf{P N}_{u a b c} \mathbf{P}^{-1}}{2 C_{m}} \mathbf{C}_{z} \Delta \mathbf{i}_{g P N 0} \\
& +\frac{\mathbf{P I}_{c a b c} \mathbf{P}^{-1}}{C_{m}}{ }_{\Delta \mathbf{n}_{u P N 0}}+\frac{\mathbf{P I}_{g a b c} \mathbf{P}^{-1}}{2 C_{m}} \Delta \mathbf{n}_{u P N 0} \\
& \frac{d \Delta \mathbf{v}_{c P N N 0}^{\Sigma}}{d t}=\frac{\mathbf{P N}_{l a b c} \mathbf{P}^{-1}}{C_{m}} \Delta \mathbf{i}_{c P N 0}-\frac{\mathbf{P N}_{l a b c} \mathbf{P}^{-1}}{2 C_{m}} \mathbf{C}_{z} \Delta \mathbf{i}_{g P N 0} \\
& +\frac{\mathbf{P} \mathbf{I}_{c a b c} \mathbf{P}^{-1}}{C_{m}} \Delta \mathbf{n}_{l P N 0}-\frac{\mathbf{C}_{m}^{-1} \mathbf{P} \mathbf{I}_{g a b c} \mathbf{P}^{-1}}{2 C_{m}} \Delta \mathbf{n}_{l P N 0}
\end{aligned}
$$

Due to the three-phase three-wire system, no zero-sequence current circulation path exists at MMC AC side. Note that in (7), $\Delta \mathbf{v}_{n}$ is the zero-sequence compensation voltage inserted into the AC side to eliminate the zero-sequence grid current in abcframe, and contains complicated multiple harmonic terms as shown in (8). To simplify the modelling, the matrix $\mathbf{C}_{z}$ is introduced to force the zero-sequence grid current zero without the need for $\Delta \mathbf{v}_{n}$, as:

$$
\mathbf{C}_{z}=\left[\begin{array}{lll}
1 & 0 & 0 \\
0 & 1 & 0 \\
0 & 0 & 0
\end{array}\right]
$$

The small-signal model of the three-phase MMC around an operation trajectory in $\mathrm{PNO}$-frame, characterized by $\triangle \mathbf{i}_{C P N O}$, $\Delta \dot{\mathbf{i}}_{g P N O}, \Delta \mathbf{v}_{\text {cuPN0 }}^{\Sigma}$ and $\Delta \mathbf{v}_{\text {clPNO }}^{\Sigma}$, can be derived in matrix form as

$$
\dot{\Delta}_{P N 0}=\mathbf{A}_{s} \Delta \mathbf{x}_{P N 0}+\mathbf{M}_{\triangle \mathbf{n}_{P N 0}}+\mathbf{B}_{\Delta \mathbf{v}_{p P N 0}}
$$

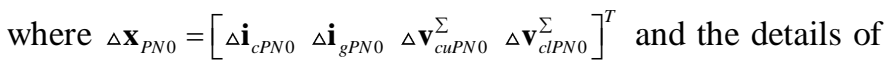
the matrices derived are:

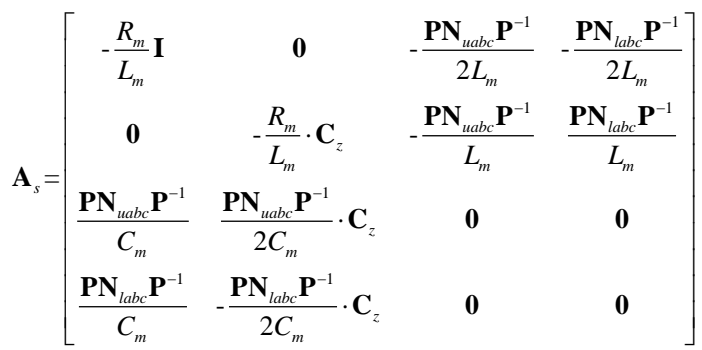

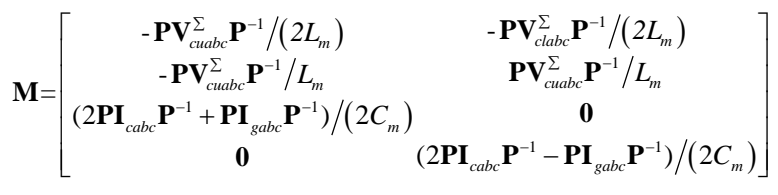

$$
\begin{aligned}
& \mathbf{B}=\left[\begin{array}{llll}
\mathbf{0} & -2 \mathbf{I} / L_{m} & \mathbf{0} & \mathbf{0}
\end{array}\right]^{T}, \Delta \mathbf{n}_{P N 0}=\left[\begin{array}{ll}
\Delta \mathbf{n}_{u P N 0} & \Delta \mathbf{n}_{I P N 0}
\end{array}\right]^{T}
\end{aligned}
$$

In order to derive the system state equation, the relationship among the modulation ratio $\Delta \mathbf{n}_{P N O}$, the state variable $\Delta \mathbf{x}_{P N O}$, and 

is subject to Institution of Electrical and Electronic Engineering Copyright. The copy of record is available at IEEE Xplore Digital Library.

the input variable $\triangle \mathbf{v}_{p P N O}$ need be identified in PNO-frame. When MMC controllers are considered, the variation of the modulation ratio $\Delta \mathbf{n}_{P N O}$ depends on the control variables of the controllers. The control variables of the MMC generally include the AC current and voltage, as well as the internal circulating current. Thus, the small signal upper and low arm modulation ratios can be expressed as:

$$
\left\{\begin{aligned}
& \Delta \mathbf{n}_{u P N 0}=-\mathbf{G}_{i P N 0} \cdot \Delta \mathbf{i}_{g P N 0}-\mathbf{G}_{v P N 0} \cdot \Delta \mathbf{V}_{g P N 0}-\mathbf{G}_{c c P N 0} \cdot \Delta \mathbf{i}_{c P N 0} \\
& \Delta \mathbf{n}_{l P N 0}=\mathbf{G}_{i P N 0} \cdot \Delta \mathbf{i}_{g P N 0}+\mathbf{G}_{v P N 0} \cdot \Delta \mathbf{V}_{g P N 0}-\mathbf{G}_{c c P N 0} \cdot \Delta \mathbf{i}_{c P N 0}
\end{aligned}\right.
$$

where $\mathbf{G}_{i P N 0}, \mathbf{G}_{v P N 0}$ and $\mathbf{G}_{c c P N 0}$ are the gain matrices of the AC current, AC voltage, and circulating current in the PNO-frame, respectively.

Rewriting (13) in matrix form yields the relationship among the modulation ratio $\Delta \mathbf{n}_{P N O}$, the state variable $\Delta \mathbf{x}_{P N O}$, and the voltage $\triangle \mathbf{v}_{P P N O}$ as:

$$
{ }_{\triangle} \mathbf{n}_{P N 0}=\mathbf{G}_{A} \cdot \triangle \mathbf{x}_{P N 0}+\mathbf{G}_{B} \cdot \Delta \mathbf{v}_{p P N 0}
$$

where $\mathbf{G}_{A}=\left[\begin{array}{cccc}-\mathbf{G}_{c c P N 0} & -\mathbf{G}_{i P N 0} & \mathbf{0} & \mathbf{0} \\ -\mathbf{G}_{c c P N 0} & \mathbf{G}_{i P N 0} & \mathbf{0} & \mathbf{0}\end{array}\right]$ and $\mathbf{G}_{B}=\left[\begin{array}{c}-\mathbf{G}_{v P N 0} \\ \mathbf{G}_{v P N 0}\end{array}\right]$.

Substituting (14) into (12) derives the small-signal statespace equation of the three-phase MMC in PNO-frame as:

$$
\dot{\mathbf{x}}_{P N 0}=\left(\mathbf{A}_{s}+\mathbf{M G}_{\mathbf{A}}\right) \Delta \mathbf{x}_{P N 0}+\left(\mathbf{B}+\mathbf{M G}_{\mathbf{B}}\right)_{\triangle \mathbf{v}_{p P N 0}}
$$

\section{MMC small-signal model based on HSS}

All the state variables in (15) are periodic signals in steadystate, and the MMC is essentially a time-periodic system, i.e., the matrices $\mathbf{A}_{\mathrm{s}}, \mathbf{B}, \mathbf{M}, \mathbf{G}_{\mathbf{A}}$ and $\mathbf{G}_{\mathbf{B}}$ are periodic [16]. Based on the HSS modelling method [12], the MMC time-domain statespace model (15) is transformed to the small-signal HSS model in frequency-domain to obtain a linear time-invariant (LTI) system expressed as:

$$
\begin{aligned}
s_{\triangle} \mathbf{X}_{P N 0} & =\left(\Gamma\left[\mathbf{A}_{s}\right]+\Gamma[\mathbf{M}] \cdot \mathbf{H G}_{A}-\mathbf{Q}\right) \cdot \Delta \mathbf{X}_{P N 0} \\
& +\left(\Gamma[\mathbf{B}]+\Gamma[\mathbf{M}] \cdot \mathbf{H G}_{B}\right) \cdot \Delta \mathbf{V}_{p P N 0}
\end{aligned}
$$

where $\Gamma\left[\mathbf{A}_{s}\right], \Gamma[\mathbf{B}]$ and $\Gamma[\mathbf{M}]$ are Toeplitz matrices. $\mathbf{H G}_{A}$ is the control transfer matrix associated with the harmonic state variables, $\mathbf{H G}_{B}$ is the one with the harmonic input variables at different frequencies, and their specific expressions are decided by the controller. $\triangle \mathbf{X}_{P N O}$ and $\Delta \mathbf{V}_{P P N O}$ are the harmonic state variable matrices and the input matrix in harmonic frequency, respectively. The expressions of $\Gamma\left[\mathbf{A}_{s}\right], \Gamma[\mathbf{B}], \Gamma[\mathbf{M}], \mathbf{H G}_{A}$,

$\mathbf{H G}_{B}, \mathbf{Q}, \Delta \mathbf{X}_{P N O}$ and $\Delta \mathbf{V}_{p P N O}$ are given in the Appendix.

To establish a complete small-signal MMC model, it is necessary to include various controllers. In (16), $\mathbf{H G}_{A}$ and $\mathbf{H} \mathbf{G}_{B}$ are the transfer function matrices determined by the controller in PNO-frame. Therefore, to derive the small-signal impedance of MMC, the transfer functions of specific controllers should be established in the actual frame where they are implemented and then transformed to PNO-frame. This enables different MMC controllers, which are usually implemented in different frames, e.g., PR circulating current controller in abc-frame and PI AC current controller in dq-frame, to be accurate modelled in the PNO-frame. The detailed procedures to determine the transfer function matrices $\mathbf{H G}_{A}$ and $\mathbf{H G}_{B}$ in the small-signal model are described in the following subsections.

\section{Circulating current suppression controller (CCSC)}

The circulating current predominantly contains a series of even harmonics, in which the second-order harmonic dominates
[19]. The objective of CCSC is to suppress the circulating current as Fig. 2 shows a typical implementation in in which 3 PR controller tuned at double fundamental frequency $\left(2 \omega_{0}\right)$ are used, one for each phase.

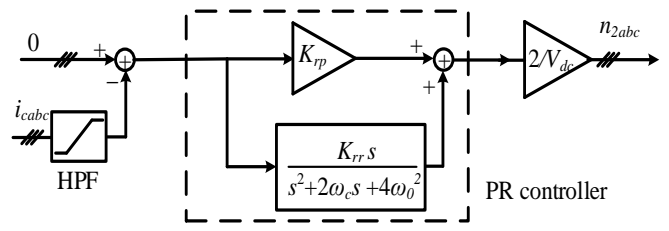

Fig. 2 Diagram of circulating current suppression controller

The transfer function of the PR controller is [27]

$$
G_{P R}(s)=K_{r p}+\frac{K_{r r}}{s^{2}+2 \omega_{c} s+4 \omega_{0}^{2}}
$$

where $K_{r p}$ and $K_{r r}$ are the proportional and resonant coefficients of the PR controller, respectively. $\omega_{c}$ is the cutoff frequency.

The high pass filter (HPF) filters out the DC component in the common mode current, and its transfer function is:

$$
G_{H P F}(s)=\frac{s^{2}}{s^{2}+2 \zeta \omega_{n} s+\omega_{n}^{2}}
$$

where $\omega_{n}$ is the un-damped natural frequency and $\zeta$ is the damping factor [30].

Thus, the double frequency output modulation signal by the CCSC and the circulating current have the following relationship:

$$
\left[\begin{array}{l}
\Delta n_{2 a} \\
\Delta n_{2 b} \\
\Delta n_{2 c}
\end{array}\right]=\mathbf{G}_{c c a b c}(s)\left[\begin{array}{c}
\Delta i_{c a} \\
\Delta i_{c b} \\
\Delta i_{c c}
\end{array}\right]
$$

where $\mathbf{G}_{c c a b c}(s)$ is the circulating current transfer function matrix in abc-frame, and is given as:

$\mathbf{G}_{c c a b c}(s)=\frac{-2}{V_{d c}}\left[\begin{array}{ccc}G_{H P F}(s) G_{P R}(s) & 0 & 0 \\ 0 & G_{H P F}(s) G_{P R}(s) & 0 \\ 0 & 0 & G_{H P F}(s) G_{P R}(s)\end{array}\right]$

The corresponding CCSC transfer function in PNO-frame $\mathbf{G}_{\mathrm{ccPN} 0}(s)$, as part of $\mathbf{H G}_{\mathrm{A}}$ in (16), can be derived as:

$$
\mathbf{G}_{c c P N 0}(\mathrm{~s})=\mathbf{P} \cdot \mathbf{G}_{c c a b c}(\mathrm{~s}) \cdot \mathbf{P}^{-1}
$$

\section{E. AC terminal current controller}

The AC terminal current control loop is typically implemented in dq-frame fixed to the voltage $\mathbf{V}_{\mathbf{g}}$ at converter AC connection point and its block diagram is presented in Fig. 3 , together with the PLL. The output of the current control loop is the fundamental frequency modulation ratio $n_{1 \mathrm{abc}}$.

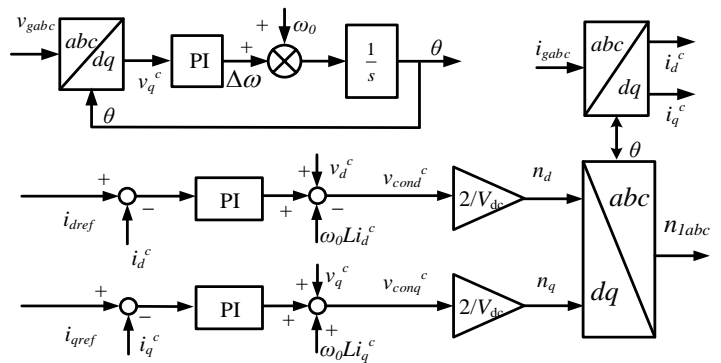

Fig. 3 The block diagram of an inner current loop

When voltage perturbation occurs, the dynamics of the PLL can be described as [28]:

$$
\Delta \theta=G_{p l l}(s) \Delta v_{q}
$$



is subject to Institution of Electrical and Electronic Engineering Copyright. The copy of record is available at IEEE Xplore Digital Library.

where $G_{p l l}(s)$ is the transfer function of the PLL expressed as:

$$
G_{p l l}(s)=\frac{K_{p p l l} s+K_{i p l l}}{s^{2}+V_{d} K_{p p l l} s+V_{d} K_{i p l l}}
$$

where $K_{\text {ppll }}$ and $K_{\text {ipll }}$ are the proportional and integral coefficients of the PLL's PI controller, respectively, and $V_{d}$ is the steady state d-axis network voltage.

In steady-state, the measured network voltages at the MMC connection point $V_{d}^{c}$ and $V_{q}^{c}$ in the control frame determined by the PLL equal to the corresponding $V_{d}$ and $V_{q}$ in the actual system frame, and can be written as [28]:

$$
\left[\begin{array}{l}
V_{d}^{c} \\
V_{q}^{c}
\end{array}\right]=\left[\begin{array}{cc}
\cos (0) & \sin (0) \\
-\sin (0) & \cos (0)
\end{array}\right]\left[\begin{array}{l}
V_{d} \\
V_{q}
\end{array}\right]
$$

However, according to (22), voltage perturbation $\Delta v_{q}$ at the connection point leads to angle deviation $\Delta \theta$ extracted by the PLL, which affects the frame transformation. For small angle deviation $\Delta \theta$, the trigonometry functions $\sin (\Delta \theta)$ and $\cos (\Delta \theta)$ are approximated to 0 and 1 in the frame transformation, respectively. Based on (22) and (24), the voltage perturbations $\Delta v_{d}$ and $\Delta v_{q}$ in system dq-frame passing through the PLL yield the voltage perturbations in the control frame as:

$$
\left[\begin{array}{c}
\Delta v_{d}^{c} \\
\Delta v_{q}^{c}
\end{array}\right]=\left[\begin{array}{cc}
1 & V_{q} G_{p l l} \\
0 & 1-V_{d} G_{p l l}
\end{array}\right]\left[\begin{array}{l}
\Delta v_{d} \\
\Delta v_{q}
\end{array}\right]
$$

In the same way, the resultant current perturbation in the control frame due to the PLL can be expressed as:

$$
\left[\begin{array}{c}
\Delta i_{d}^{c} \\
\Delta i_{q}^{c}
\end{array}\right]=\left[\begin{array}{cc}
0 & I_{q} G_{p l l} \\
0 & -I_{d} G_{p l l}
\end{array}\right]\left[\begin{array}{l}
\Delta v_{d} \\
\Delta v_{q}
\end{array}\right]+\left[\begin{array}{c}
\Delta i_{d} \\
\Delta i_{q}
\end{array}\right]
$$

where $I_{d}$ and $I_{q}$ are the d-axis and q-axis steady-state currents, respectively.

The small signal voltage references in system dq-frame can be obtained as:

$$
\left[\begin{array}{c}
\Delta v_{\text {cond }} \\
\Delta v_{\text {conq }}
\end{array}\right]=\left[\begin{array}{c}
\Delta v_{\text {cond }}^{c} \\
\Delta v_{\text {conq }}^{c}
\end{array}\right]+\left[\begin{array}{cc}
0 & -V_{\text {conq }} G_{p l l} \\
0 & V_{\text {cond }} G_{p l l}
\end{array}\right]\left[\begin{array}{l}
\Delta v_{d} \\
\Delta v_{q}
\end{array}\right]
$$

where $V_{\text {cond }}$ and $V_{\text {conq }}$ are the steady-state output d-axis and qaxis voltages of the AC current control loop, respectively.

To derive a simplified matrix form, we can define the following matrices:

$$
\begin{aligned}
& \mathbf{A}=\left[\begin{array}{cc}
1 & V_{q} G_{p l l} \\
0 & 1-V_{d} G_{p l l}
\end{array}\right], \quad \mathbf{B}=\left[\begin{array}{cc}
0 & I_{q} G_{p l l} \\
0 & -I_{d} G_{p l l}
\end{array}\right], \quad \mathbf{C}=\left[\begin{array}{cc}
G_{i P I} & 0 \\
0 & G_{i P I}
\end{array}\right], \\
& \mathbf{D}=\left[\begin{array}{cc}
0 & -\omega_{0} L_{m} \\
\omega_{0} L_{m} & 0
\end{array}\right], \text { and } \mathbf{E}=\left[\begin{array}{cc}
0 & -V_{\text {conq }} G_{p l l} \\
0 & V_{\text {cond }} G_{p l l}
\end{array}\right],
\end{aligned}
$$

where $G_{i P I}$ is the transfer function of the current PI controller. According to the structure of the current loop shown in Fig. 3, the perturbations of the modulation ratios are determined by the perturbations of the voltage and current in dq-frame as:

$$
\Delta \mathbf{n}_{d q}=\mathbf{G}_{i d q} \Delta \mathbf{i}_{d q}+\mathbf{G}_{v d q} \Delta \mathbf{v}_{d q}
$$

where

$$
\begin{gathered}
\mathbf{G}_{i d q}=2(\mathbf{D}-\mathbf{C}) / V_{d c} \\
\mathbf{G}_{v d q}=2(\mathbf{D B}+\mathbf{E}+\mathbf{A}-\mathbf{C B}) / V_{d c}
\end{gathered}
$$

\section{F. Outer-loop controller}

The outer-loop controller is designed to set the current reference $i_{\text {dref }}$ and $i_{\text {qref }}$ for the inner-loop AC current controller. Fig. 4 shows typical outer loop control designs with active and reactive power control (PQ control), and active power and $\mathrm{AC}$ voltage control (PV control).
As shown in Fig. 4, for AC voltage control, the linearised terminal voltage magnitude of $\mathrm{MMC}$ is expressed as:

$$
\Delta v=\frac{V_{d} \Delta v_{d}^{c}+V_{q} \Delta v_{q}^{c}}{\sqrt{V_{d}^{2}+V_{q}^{2}}}
$$

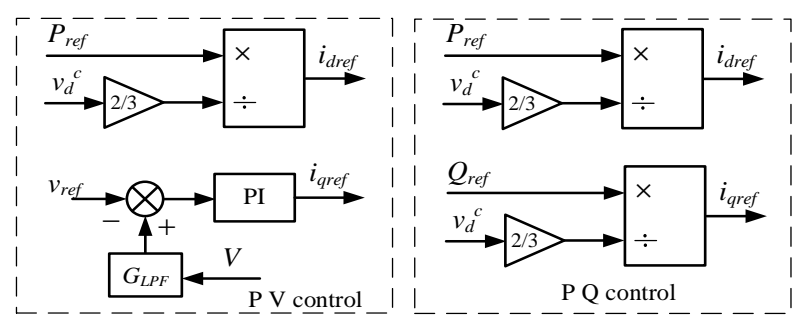

Fig. 4 Outer-loop: PV and PQ control

For the active and reactive power control, the linearised dand q-axis current references can be obtained as:

$$
\begin{gathered}
\Delta i_{\text {dref }}=-2 P_{r e f} \Delta v_{d}^{c} /\left(3 V_{d}^{2}\right) \\
\Delta i_{\text {qref }}=-2 Q_{\text {ref }} \Delta v_{d}^{c} /\left(3 V_{d}^{2}\right)
\end{gathered}
$$

Thus, with PV control and considering (31) and (32), the linearized model of PV control can be described as:

$$
\left[\begin{array}{l}
\Delta i_{\text {dref }} \\
\Delta i_{\text {qref }}
\end{array}\right]=\left[\begin{array}{cc}
1 & 0 \\
0 & G_{L P F} G_{v P I}
\end{array}\right]\left[\begin{array}{cc}
-2 P_{r e f} / 3 V_{d}^{2} & 0 \\
V_{d} / \sqrt{V_{d}^{2}+V_{q}^{2}} & V_{q} / \sqrt{V_{d}^{2}+V_{q}^{2}}
\end{array}\right]\left[\begin{array}{l}
\Delta v_{d}^{c} \\
\Delta v_{q}^{c}
\end{array}\right]
$$

where $G_{L P F}$ is the transfer function of the low pass filter in the AC voltage measurement and $G_{v P I}$ denotes the transfer function for the voltage-loop PI controller, as:

$$
G_{L P F}=\frac{1}{s T+1}, \quad G_{v P I}=K_{v p}+\frac{K_{v i}}{s}
$$

where $T$ is the time constant of the low pass filter [30], $K_{v p}$ and $K_{v i}$ are the proportional and integral coefficients of the AC voltage control loop.

$$
\text { Define } \mathbf{X}=\left[\begin{array}{cc}
1 & 0 \\
0 & G_{L P F} G_{v P I}
\end{array}\right]\left[\begin{array}{cc}
-2 P_{r e f} / 3 V_{d}^{2} & 0 \\
V_{d} / \sqrt{V_{d}^{2}+V_{q}^{2}} & V_{q} / \sqrt{V_{d}^{2}+V_{q}^{2}}
\end{array}\right] \text { and }
$$

according to Fig. 3, the corresponding $\mathbf{G}_{i d q}$ and $\mathbf{G}_{v d q}$ in (28) with the PV outer-loop controller can be derived as:

$$
\begin{aligned}
& \mathbf{G}_{i d q}=2(\mathbf{D}-\mathbf{C}) / V_{d c} \\
& \mathbf{G}_{v d q}=2(\mathbf{D B}+\mathbf{E}+\mathbf{A}-\mathbf{C B}+\mathbf{C X A}) / V_{d c}
\end{aligned}
$$

When the outer-loop adopts PQ control, combining (32) with (33) also yields the linearized transfer function of the controller in the same form as illustrated in (36) in which $\mathrm{X}$ is now given as $\mathbf{X}=\left[\begin{array}{ll}-2 P_{r e f} / 3 V_{d}^{2} & 0 \\ -2 Q_{r e f} / 3 V_{d}^{2} & 0\end{array}\right]$.

$\mathbf{G}_{i d q}$ and $\mathbf{G}_{v d q}$ are in dq-frame, and can generally be expressed as (taking $\mathbf{G}_{i d q}$ as an example)

$$
\mathbf{G}_{i d q}(s)=\left[\begin{array}{ll}
G_{i d d}(s) & G_{i d q}(s) \\
G_{i q d}(s) & G_{i q q}(s)
\end{array}\right]
$$

After transformation to the PNO-frame in a similar way as in (21), the controller transfer functions in PNO-frame become

$$
\mathbf{G}_{i P N 0}(s)=\left[\begin{array}{ccc}
G_{i P P}(s) & G_{i P N}(s) & 0 \\
G_{i N P}(s) & G_{i N N}(s) & 0 \\
0 & 0 & 0
\end{array}\right]
$$

The elements in the matrices above can be obtained as [29]: 
This paper is a post-print of a paper submitted to and accepted for publication in IEEE Journal of Emerging and Selected Topics in Power Electronics and is subject to Institution of Electrical and Electronic Engineering Copyright. The copy of record is available at IEEE Xplore Digital Library.

$\left\{\begin{array}{l}G_{i P P}(s)=\frac{1}{2}\left[G_{i d d}\left(s-j \omega_{0}\right)+G_{i q q}\left(s-j \omega_{0}\right)-j G_{i d q}\left(s-j \omega_{0}\right)+G_{i q d}\left(s-j \omega_{0}\right)\right] \\ G_{i P N}(s)=\frac{1}{2}\left[G_{i d d}\left(s+j \omega_{0}\right)-G_{i q q}\left(s+j \omega_{0}\right)+j G_{i d q}\left(s+j \omega_{0}\right)+G_{i q d}\left(s+j \omega_{0}\right)\right] \\ G_{i N P}(s)=\frac{1}{2}\left[G_{i d d}\left(s-j \omega_{0}\right)-G_{i q q}\left(s-j \omega_{0}\right)-j G_{i d q}\left(s-j \omega_{0}\right)-G_{i q d}\left(s-j \omega_{0}\right)\right] \\ G_{i N N}(s)=\frac{1}{2}\left[G_{i d d}\left(s+j \omega_{0}\right)+G_{i q q}\left(s+j \omega_{0}\right)+j G_{i d q}\left(s+j \omega_{0}\right)-G_{i q d}\left(s+j \omega_{0}\right)\right]\end{array}\right.$

According to (38) and (39), similar to 2-level VSC in [29], there exists coupling between positive and negative sequence frequencies caused by the dq-frame controller and other harmonic state variables in the PNO-frame. The matrix $\mathbf{H G}_{\mathrm{A}}$ in the Appendix need to be modified accordingly as:

$$
\mathbf{H G}_{\mathbf{A}}=\left[\begin{array}{ccccccc}
\ddots & \vdots & \vdots & \vdots & \vdots & \vdots & . \\
\cdots & \mathbf{G}_{\mathbf{A}}\left(s-j 2 \omega_{0}\right) & \mathbf{0} & \mathbf{G N P}_{\mathbf{A}}(s) & \mathbf{0} & \mathbf{0} & \cdots \\
\cdots & \mathbf{0} & \mathbf{G}_{\mathbf{A}}\left(s-j \omega_{0}\right) & \mathbf{0} & \mathbf{G N P}_{\mathbf{A}}\left(s+j \omega_{0}\right) & \mathbf{0} & \cdots \\
\cdots & \mathbf{G P N}_{\mathbf{A}}\left(s-j 2 \omega_{0}\right) & \mathbf{0} & \mathbf{G}_{\mathbf{A}}(s) & \mathbf{0} & \mathbf{G N P}_{\mathbf{A}}\left(s+j 2 \omega_{0}\right) & \cdots \\
\cdots & 0 & \mathbf{G P N}_{\mathbf{A}}\left(s-j \omega_{0}\right) & \mathbf{0} & \mathbf{G}_{\mathbf{A}}\left(s+j \omega_{0}\right) & \mathbf{0} & \cdots \\
\cdots & 0 & \mathbf{0} & \mathbf{G P N}_{\mathbf{A}}(s) & \mathbf{0} & \mathbf{G}_{\mathbf{A}}\left(s+j 2 \omega_{0}\right) & \cdots \\
\therefore & \vdots & \vdots & \vdots & \vdots & \vdots & \ddots
\end{array}\right]
$$

In (40), $\mathbf{G N P}_{\mathrm{A}}$ and $\mathbf{G P N}$ A are the frequency coupling matrices created by dq-frame controller as

$$
\mathbf{G N P}_{\mathbf{A}}(s)=\left[\begin{array}{ccccc}
\mathbf{0} & -\mathbf{G N P}_{i P N 0} & \mathbf{0} & \mathbf{0} \\
\mathbf{0} & \mathbf{G N P}_{i P N 0} & \mathbf{0} & \mathbf{0}
\end{array}\right], \mathbf{G P N}_{\mathbf{A}}(s)=\left[\begin{array}{cccc}
\mathbf{0} & -\mathbf{G P N}_{i P N 0} & \mathbf{0} & \mathbf{0} \\
\mathbf{0} & \mathbf{G P N}_{i P N 0} & \mathbf{0} & \mathbf{0}
\end{array}\right]
$$

where $\mathbf{G N P}_{i P N 0}(s)=\left[\begin{array}{ccc}0 & 0 & 0 \\ G_{i N P}(s) & 0 & 0 \\ 0 & 0 & 0\end{array}\right], \mathbf{G P N}_{i P N 0}(s)=\left[\begin{array}{ccc}0 & G_{i P N}(s) & 0 \\ 0 & 0 & 0 \\ 0 & 0 & 0\end{array}\right]$.

The matrix $\mathbf{H G}_{\mathrm{B}}$ can also be derived using the same approach.

\section{SMALL-SIGNAL ADMITTANCE OF MMC IN PNO-FRAME}

The solution of (16) can be expressed as

$$
\begin{aligned}
\Delta \mathbf{X}_{P N 0} & =\left(s \mathbf{I}-\Gamma\left[\mathbf{A}_{s}\right]-\Gamma[\mathbf{M}] \cdot \mathbf{H G}_{A}+\mathbf{Q}\right)^{-1} \cdot\left(\Gamma[\mathbf{B}]+\Gamma[\mathbf{M}] \cdot \mathbf{H G}_{B}\right) \cdot{ }_{\Delta} \mathbf{V}_{p P N 0} \\
& =\mathbf{H}_{\mathrm{hss}} \cdot \Delta \mathbf{V}_{p P N 0}
\end{aligned}
$$

where the matrix $\mathbf{H}_{\mathrm{hss}}$ reflects the relationship between the input variables $\Delta \mathbf{V}_{P P N 0}$ and state variables $\Delta \mathbf{X}_{P N 0}$. The small-signal admittance matrix of MMC $\mathbf{Y}_{M M C}$ links the AC terminal voltage and current perturbations as

$$
\Delta \mathbf{i}_{g P N}=\mathbf{Y}_{M M C} \Delta \mathbf{v}_{p P N}
$$

In PNO-frame, $\triangle \mathbf{i}_{g P N}$ is part of the state variable matrix $\Delta \mathbf{X}_{P N 0}$ and $\Delta \mathbf{v}_{p P N}$ is part of the input matrix $\Delta \mathbf{V}_{p P N O}$. Thus $\mathbf{Y}_{M M C}$ can be extracted directly from the matrix $\mathbf{H}_{\mathrm{hss}}$. Considering the harmonics in $\Delta \mathbf{i}_{g P N}$ and $\Delta \mathbf{v}_{P P N}, \mathbf{Y}_{M M C}$ will have a large dimension [18][19]. Therefore, further analysis of the MMC admittance matrix $\mathbf{Y}_{M M C}$ is required.

As for the MMC, the even harmonics in the upper and the lower arms of any phase have the same magnitude and phase (called a common-mode (CM) harmonic), while the same magnitude but $180^{\circ}$ phase difference (called a differentialmode (DM) harmonic) for odd harmonics [31]. The CM components circulate in the internal MMC while the DM components ouput through the MMC AC terminals.

If a positive-sequence perturbation $\Delta \mathbf{v}_{p a b c}$ at $\omega_{p}$ appears at the MMC AC terminal, the upper and lower arm equivalent capacitor $\mathrm{C}_{\mathrm{m}}$ will have the positive-sequence response voltage $\Delta \mathbf{v}_{\text {cuabc }}^{\Sigma}$ and $\Delta \mathbf{v}_{\text {clabc }}^{\Sigma}$ at $\omega_{p}$, respectively. Because the upper and lower arms are symmetrical, the perturbation voltage $\Delta \mathbf{v}_{\text {cuabc }}^{\Sigma}$, $\Delta \mathbf{v}_{\text {clabc }}^{\Sigma}$ belongs to the DM components, i.e., the same magnitude but $180^{\circ}$ phase difference. Taking the positive-sequence capactior voltage perturbations $\Delta \mathbf{v}_{\text {cuabc }}^{\Sigma}, \Delta \mathbf{v}_{\text {clabc }}^{\Sigma}$ for the upper and lower arms as an example, they may be expressed as:

$$
\Delta \mathbf{v}_{\text {cuabc }}^{\Sigma}=-\Delta \mathbf{v}_{\text {clabc }}^{\Sigma}=\left[\begin{array}{c}
\Delta m_{c} \cos \left(\omega_{p} t+\Delta \theta_{c}\right) \\
\Delta m_{c} \cos \left(\omega_{p} t+\Delta \theta_{c}-2 \pi / 3\right) \\
\Delta m_{c} \cos \left(\omega_{p} t+\Delta \theta_{c}+2 \pi / 3\right)
\end{array}\right]
$$

where $\Delta m_{c}$ and $\Delta \theta_{c}$ are the magnitude and the phase angle of the perturbation voltage, respectively.

The steady-state values of the modulation ratio for the upper and lower arms are $\mathbf{N}_{u a b c}$ and $\mathbf{N}_{l a b c}$, mainly including the DC, fundamental and double-frequency $(h=2)$ components. The impact of the different components on the MMC AC terminal current are now considered.

- For the DC components of $\mathbf{N}_{u a b c}$ and $\mathbf{N}_{l a b c}$, both $\mathbf{N}_{\text {uabc } 0} \Delta \mathbf{v}_{\text {cuabc }}^{\Sigma}$ and $\mathbf{N}_{\text {labc } 0} \Delta \mathbf{V}_{\text {clabc }}^{\sum}$ are positive-sequence variables with the same frequency $\omega_{p}$ but opposite sign, resulting in positive-sequence voltage at $\omega_{p}$ generated at the MMC terminal. Consequently, positive-sequence current at $\omega_{p}$ is generated at the MMC AC terminal.

- For the fundamental frequency components of $\mathbf{N}_{u a b c}$ and $\mathbf{N}_{l a b c}$, they are DM components and $\mathbf{N}_{u a b c 1}=-\mathbf{N}_{l a b c 1}$. Thus, $\mathbf{N}_{u a b c 1} \Delta \mathbf{V}_{\text {cuabc }}^{\Sigma}$ equals $\mathbf{N}_{\text {labc1 }} \Delta \mathbf{V}_{\text {clabc }}^{\Sigma}$, and the two appear as MMC internal CM components. Thus, no current or voltage response at the MMC AC terminal will be observed.

- For the double-frequency components of $\mathbf{N}_{u a b c 2}$ and $\mathbf{N}_{l a b c 2}$, they are the CM components and identical as:

$$
\begin{aligned}
& \mathbf{N}_{\text {uabc } 2}=\mathbf{N}_{\text {labc } 2} \\
& =\left[\begin{array}{lll}
N_{2} \cos \left(2 \omega_{0} t+\theta_{2}\right) & & \\
& N_{2} \cos \left(2 \omega_{0} t+\theta_{2}+2 \pi / 3\right) & \\
& & N_{2} \cos \left(2 \omega_{0} t+\theta_{2}-2 \pi / 3\right)
\end{array}\right]
\end{aligned}
$$

The product of the perturbation arm capacitor voltage $\Delta \mathbf{V}_{\text {cuabc }}^{\Sigma}$ and $\mathbf{N}_{\text {uabc2 }}$ is:

$\mathbf{N}_{\text {uabc } 2} \Delta \mathbf{V}_{\text {cuabc }}^{\Sigma}=-\mathbf{N}_{\text {labc } 2} \Delta \mathbf{v}_{\text {clabc }}^{\Sigma}$

$=\frac{\Delta m_{c} N_{2}}{2}\left[\begin{array}{c}\cos \left[\left(\omega_{p}+2 \omega_{0}\right) t+\left(\Delta \theta_{c}+\theta_{2}\right)\right]+\cos \left[\left(\omega_{p}-2 \omega_{0}\right) t+\left(\Delta \theta_{c}-\theta_{2}\right)\right] \\ \cos \left[\left(\omega_{p}+2 \omega_{0}\right) t+\left(\Delta \theta_{c}+\theta_{2}\right)\right]+\cos \left[\left(\omega_{p}-2 \omega_{0}\right) t+\left(\Delta \theta_{c}-\theta_{2}\right)+2 \pi / 3\right] \\ \cos \left[\left(\omega_{p}+2 \omega_{0}\right) t+\left(\Delta \theta_{c}+\theta_{2}\right)\right]+\cos \left[\left(\omega_{p}-2 \omega_{0}\right) t+\left(\Delta \theta_{c}-\theta_{2}\right)-2 \pi / 3\right]\end{array}\right]$

According to (46), the interaction between the two yields zero-sequence voltages at $\omega_{p}+2 \omega_{0}$ with opposite direction for the upper and lower arms. For a three-wire system, such zero-sequence voltage only exists in the internal $\mathrm{MMC}$, and there is no zero-sequence current or voltage at $\omega_{p}+2 \omega_{0}$ on the AC terminal. However, the generated negative-sequence voltages at $\omega_{p}-2 \omega_{0}$ for the upper and lower arms are DM components, and hence, will appear at the AC terminal with the corresponding current.

- Similarly to $h=2$, with $h=4$, there exists only $\omega_{p}+4 \omega_{0}$ at the MMC terminal but can be neglected due to its very small magnitude. Whereas for $h>4$, the $h$-th harmonics in the MMC are all very small and the response at $\omega_{p} \pm h \omega_{0}$ can be ignored.

Therefore, based on the above observation, the specific form of the small-signal admittance $\mathbf{Y}_{\text {MMC }}$ at the MMC terminal can be simplified as a 2 by 2 matrix expressed

$$
\left[\begin{array}{c}
\Delta i_{g P}(s) \\
\Delta i_{g N}\left(s-j 2 \omega_{0}\right)
\end{array}\right]=\left[\begin{array}{cc}
Y_{P P}(s) & Y_{P N}(s) \\
Y_{N P}\left(s-j 2 \omega_{0}\right) & Y_{N N}\left(s-j 2 \omega_{0}\right)
\end{array}\right]\left[\begin{array}{c}
\Delta v_{p P}(s) \\
\Delta v_{p N}\left(s-j 2 \omega_{0}\right)
\end{array}\right]
$$

where $Y_{P P}(s), Y_{P N}(s), Y_{N P}\left(s-j 2 \omega_{0}\right)$, and $Y_{N N}\left(s-j 2 \omega_{0}\right)$ are the four elements extracted from the matrix $\mathbf{H}_{\mathrm{hss}}$. 
This paper is a post-print of a paper submitted to and accepted for publication in IEEE Journal of Emerging and Selected Topics in Power Electronics and is subject to Institution of Electrical and Electronic Engineering Copyright. The copy of record is available at IEEE Xplore Digital Library.

Thus, the form of the MMC admittance is effectively a 2 by 2 matrices and hence the system stability analysis can be carried out by application of the Generalised Nyquist Criterion.

It is noted that the MMC admittance as indicated in (42) and (43) is depended on the operating point and therefore, different operating points will result in different MMC admittances.

\section{MODEL VALIDATION AND STABILITY ASSESSMENT}

In order to validate the developed HSS model, the admittance plots from the HSS model are compared to those obtained from corresponding time-domain models using frequency sweep method. The time-domain models are implemented in Matlab/Simulink and the HSS model as described in this section, is implemented by using an m.file in Matlab. The main electrical parameters of the MMC system are listed in Table I. In the time-domain models, the small-signal impedance of the MMC is measured by means of injecting a series of small positive and negative-sequence perturbations $\Delta v_{p a}, \Delta v_{p b}$, and $\Delta v_{p c}$ as shown in Fig. 1, of which the peak phase voltage is $3 \mathrm{kV}$ at different frequencies. The AC current response $\Delta i_{g a}, \Delta i_{g b}$, and $\Delta i_{g c}$ of the MMC system under each frequency is measured and the admittance under this frequency is calculated using (47).

\begin{tabular}{|c|c|} 
Table I Main electrical parameters of the MMC system \\
\hline Parameters & Value \\
\hline Rated active and reactive power $(\mathrm{P}, \mathrm{Q})$ & $1000 \mathrm{MW}, \pm 300 \mathrm{MVar}$ \\
\hline Nominal DC Voltage $\left(\mathrm{V}_{\mathrm{dc}}\right)$ & $\pm 320 \mathrm{kV}$ \\
\hline Rated MMC AC voltage $(\mathrm{L}-\mathrm{L})\left(\mathrm{V}_{\mathrm{nl}}\right)$ & $360 \mathrm{kV}$ \\
\hline Arm resistance and inductance $\left(\mathrm{R}_{\mathrm{m}} \mathrm{L}_{\mathrm{m}}\right)$ & $0.08 \Omega, 0.042 \mathrm{H}$ \\
\hline Lumped cell capacitance $\left(\mathrm{C}_{\mathrm{m}}\right)$ & $31.4 \mu \mathrm{F}$ \\
\hline Nominal Frequency $\left(\mathrm{f}_{0}\right)$ & $50 \mathrm{~Hz}$ \\
\hline Transformer rated apparent power $(\mathrm{St})$ & $1265 \mathrm{MVA}$ \\
\hline Transformer voltage ratio $(\mathrm{kt})$ & $400 / 360 \mathrm{kV}$ \\
\hline Transformer leakage reactance $\mathrm{Xt} *$ & $0.18 \mathrm{pu}$ \\
\hline
\end{tabular}

\section{A. Admittance analysis from time-domain model}

Initial tests in the time domain model with the MMC under open-loop control is carried out. The 3-phase modulation ratio for the arms are assigned directly, e.g., for phase 'a' upper arm, $n_{\text {uа }}=0.5-0.46\left[\cos \left(\omega_{0} t+0.07\right)\right]+0.01\left[\cos \left(2 \omega_{0} t+0.07\right)\right] . \quad$ Voltage perturbations of $40 \mathrm{~Hz}$ positive and negative sequence are injected at the MMC AC terminal, separately. FFT analysis is conducted on the phase ' $a$ ' current and voltage and selected spectra are shown in Fig. 5 in which the $50 \mathrm{~Hz}$ fundamental frequency components have been omitted for clarity.

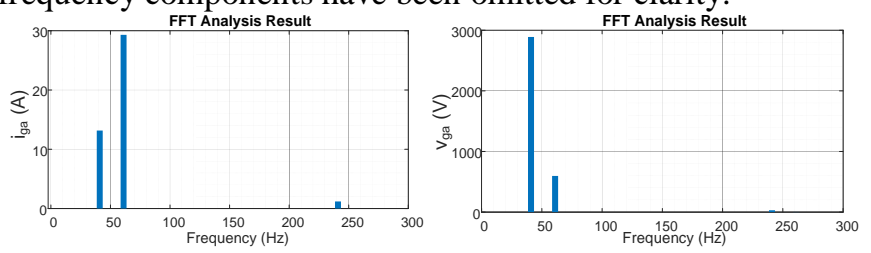

(a) With $40 \mathrm{~Hz}$ positive-sequence voltage injection

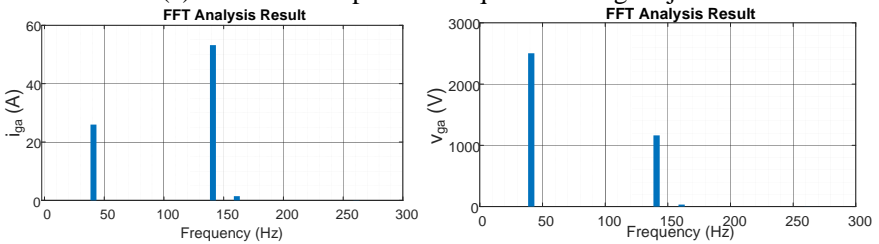

(b) With $40 \mathrm{~Hz}$ negative-sequence voltage injection

Fig. 5 FFT results of AC terminal current and voltage $\left(i_{g a}, v_{g a}\right)$ with voltage perturbation injection.

Table II Phase angles of the 3-phase voltage and current with $40 \mathrm{~Hz}$ positive and negative sequence voltage injections (degree)

\begin{tabular}{|c|c|c|c|c|c|c|}
\hline & \multicolumn{3}{|c|}{ Positive sequence $40 \mathrm{~Hz}$} & \multicolumn{3}{c|}{ Negative sequence $40 \mathrm{~Hz}$} \\
\cline { 2 - 7 } & $40 \mathrm{~Hz}$ & $60 \mathrm{~Hz}$ & $240 \mathrm{~Hz}$ & $40 \mathrm{~Hz}$ & $140 \mathrm{~Hz}$ & $160 \mathrm{~Hz}$ \\
\hline$\Delta v_{g a}$ & 85.3 & -63.5 & 71.9 & 93.6 & 80.7 & 241.1 \\
\hline$\Delta v_{g b}$ & -34.7 & 176.5 & 191.9 & 213.6 & -39.3 & 1.1 \\
\hline$\Delta v_{g c}$ & 205.3 & 56.5 & -48.1 & -26.4 & 200.7 & 121.1 \\
\hline$\Delta i_{g a}$ & 150.4 & 105.9 & 214.9 & 64.5 & 236.9 & 34.4 \\
\hline$\Delta i_{g b}$ & 30.4 & -14.1 & -25.1 & 184.5 & 116.9 & 154.4 \\
\hline$\Delta i_{g c}$ & -89.6 & 225.9 & 94.9 & -55.5 & -3.1 & -85.6 \\
\hline
\end{tabular}

Fig. 5 (a) shows that under $40 \mathrm{~Hz}$ positive-sequence voltage perturbation, there are multiple frequency responses in the voltage and current at $40 \mathrm{~Hz}, 60 \mathrm{~Hz}$ and $240 \mathrm{~Hz}$. Table II shows the phase angles for the voltage and current responses. It can be observed that:

- The voltage and current responses are positive-sequence at $40 \mathrm{~Hz}$ and $60 \mathrm{~Hz}$, and negative-sequence at $240 \mathrm{~Hz}$.

- The resulted positive-sequence response at $60 \mathrm{~Hz}$ can also be considered as negative-sequence at $-60 \mathrm{~Hz}$, as $-60 \mathrm{~Hz}$ negative-sequence indicates $60 \mathrm{~Hz}$ positive-sequence in time-domain [32].

- Thus, it can be concluded that the injected positivesequence voltage perturbation at $\omega_{p}$ leads to a positivesequence response at $\omega_{p}$ and negative-sequence responses at $\omega_{p}-2 \omega_{0}$ and $\omega_{p}+4 \omega_{0}$, though the negative-sequence response at $\omega_{p}+4 \omega_{0}$ is very small.

For $40 \mathrm{~Hz}$ negative-sequence voltage perturbation, Fig. 5 (b) shows the voltage and current responses at $40 \mathrm{~Hz}, 140 \mathrm{~Hz}$ and $160 \mathrm{~Hz}$, in which the response at $160 \mathrm{~Hz}$ is negligible. Table II shows the corresponding voltage and current the phase angles. It can be observed that:

- The response is negative-sequence at $40 \mathrm{~Hz}$, positivesequence at $140 \mathrm{~Hz}$, and negative-sequence at $160 \mathrm{~Hz}$.

- According to the analysis in Section IV, the negativesequence input at $\omega_{p}$ causes the negative-sequence at $\omega_{p}$ $(40 \mathrm{~Hz})$ and positive-sequence response at $\omega_{p}+2 \omega_{0}(140 \mathrm{~Hz})$ and $\omega_{p}-4 \omega_{0}(-160 \mathrm{~Hz})$.

- Positive-sequence $-160 \mathrm{~Hz}$ is deemed negative-sequence at $160 \mathrm{~Hz}$ in time-domain.

The above simulation results verify the theoretical analysis in Section IV, and the small-signal model of MMC in PNOframe is properly captured by the four admittance elements in (47).

\section{B. Admittance validation}

Fig. 6 compares the admittance elements $Y_{P P}(s)$ and $Y_{P N}(s)$ in matrix $\mathbf{Y}_{\text {MMC }}$ derived from the HSS model with different harmonic orders considered, and those obtained from the timedomain model. The MMC exports 1000MW / 0MVar to the AC grid and the AC terminal voltage is $1 \mathrm{pu}$. Open-loop control is considered and the 3-phase modulations for the arms are the same as in Section IV A. The other two elements in $\mathbf{Y}_{\mathbf{M M C}}$, $Y_{N P}\left(s-2 j \omega_{0}\right)$, and $Y_{N N}\left(s-2 j \omega_{0}\right)$, have similar trends and due to space limit, are not presented here. Comparing the different admittance curves, it can be found that higher harmonic order considered in the analytical HSS model leads to more accurate model, and for $h=4$ the analytical admittances match well with those of the time-domain simulation models. It also implies that the internal harmonics of MMC has a significant impact on the AC side small-signal admittance, and need to be considered in the modelling. 
When the complete control including CCSC, AC current loop, PLL and outer-loop are included, Fig. 7 compares the smallsignal MMC admittances obtained from the time-domain model and the HSS model $(h=4)$. Due to space limitation, only the positive admittance $Y_{P P}(s)$ is presented here. As can be seen that the results from the two models match well under both PV and PQ control, while the outer loop also has a significant impact on MMC impedance. In the case of PQ control, the magnitude of $Y_{P P}$ at $50 \mathrm{~Hz}-70 \mathrm{~Hz}$ is smaller than that in PV control, which contributes to improved system stability [33]. Moreover, the phase of $Y_{P P}$ with PQ control is closer to 0 than that with PV control and thus has larger damping with better stability. Under both controls, the phase of $Y_{P P}$ among $90-270^{\circ}$ at $50-70 \mathrm{~Hz}$ implies the negative resistance effect and potentially leads to oscillation [34]. In this scenario, the PQ control is better than PV control. For frequencies above $70 \mathrm{~Hz}$, the admittances under the two controls are almost the same and the phase is between $90^{\circ}$ and $90^{\circ}$.
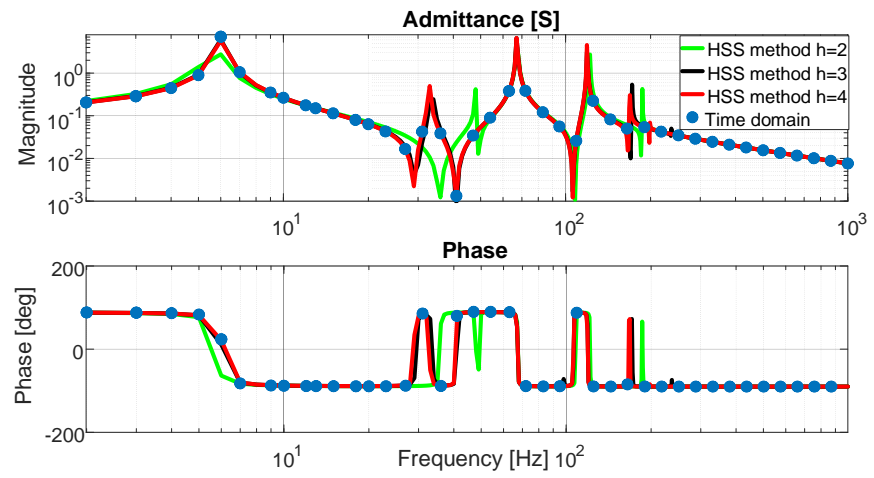

(a)
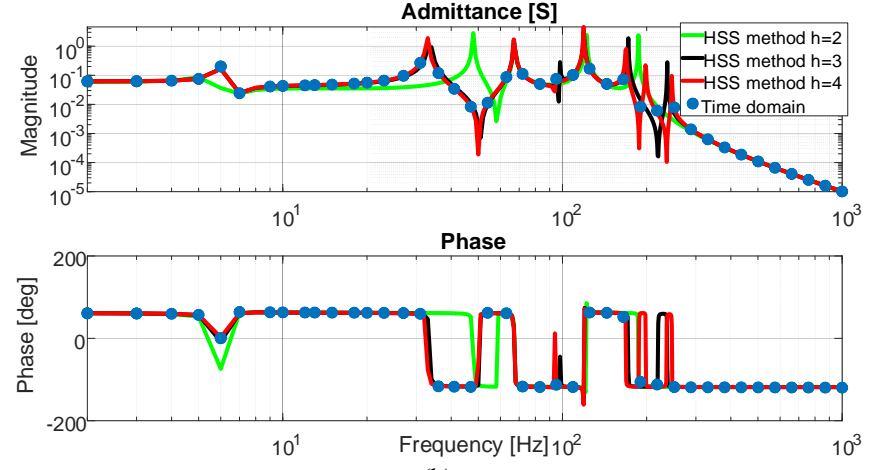

(b)

Fig. 6 Validation of the MMC admittance. (a) $Y_{P P}(s)$, (b) $Y_{P N}(s)$
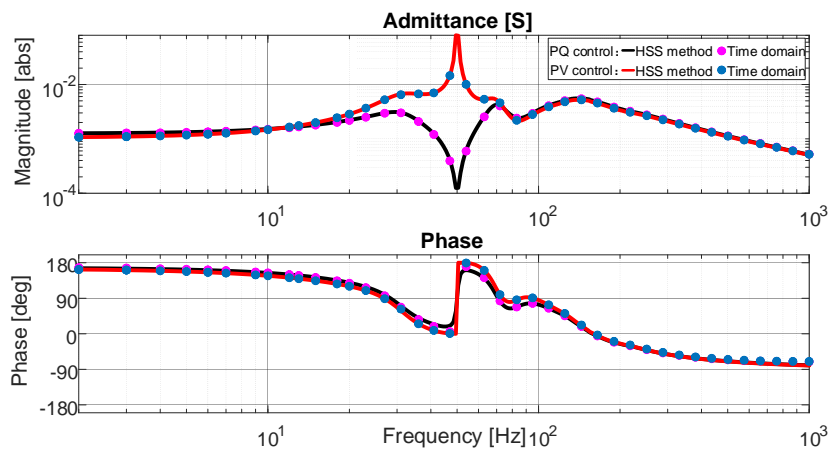

Fig. 7 Positive admittance $Y_{P P}(s)$ with different out-loop controllers

Comparing $Y_{P P}(s)$ in Fig. 6 (a) and Fig. 7, it can be seen that the resonant points are much reduced owing to the internal harmonic decline when the full controllers are added in the system.

The analytical model also reveals that the impedance of the MMC is highly dependent on control structure and parameter setting, system operating point etc. The obtained MMC impedances during various system settings and conditions have also been validated but due to space limitation, they are not shown here.

\section{STABILITY OF CONVERTERS IN CLOSE PROXIMITY}

\section{A. Equivalent network for multi-converter connection}

Considering increased network interconnections and connection of large offshore wind farms (e.g. in Europe) using HVDC links, many power networks in Europe will see significant numbers of HVDC converters connected in close proximity. For example, as outlined in [35] and schematically shown in Fig. 8, the GB network will have more than 20 HVDC connection by 2027 , with a total transmission capacity of over $16 \mathrm{GW}$. When multiple converters are considered for studying converter interaction, shown in the red area in Fig. 8 as an example, the network admittance seen at each of the converter connection points will need to be considered together with the electrical coupling between the converters. In order to perform an analytical study on system stability and interaction, a simplified network structure is required. In this paper, the socalled multi-infeed interaction factor (MIIF) between the converters [36], proposed by CIGRE WG B4 is used to quantify the simplified system structures. MIIF is a parameter for estimating the degree of voltage interaction between converters. Converter AC busses electrically far apart will have low MIIF, while MIIF is high when the AC busses are very close and the interaction is strong between the converters. The general formula for calculating $M I I F_{e, n}$ is expressed as [36]

$$
M I I F_{e, n}=\Delta V_{e} / \Delta V_{n}
$$

where $\Delta V_{e}$ is the observed voltage change at bus $e$ when a small voltage change $\Delta V_{n}$ is induced at bus $n$. MIIF values range from zero to one with zero implying infinite electrical separation between $e$ and $n$ and one being on the same bus.

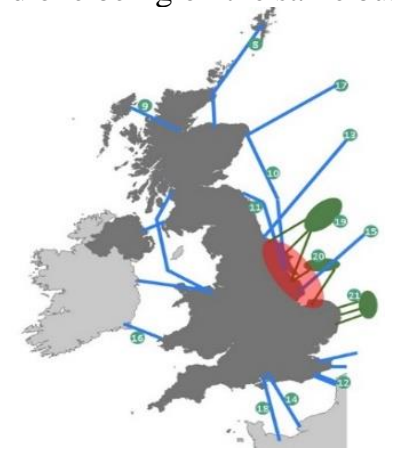

Fig. 8 A future outlook of HVDC connection in the GB power grid [35]

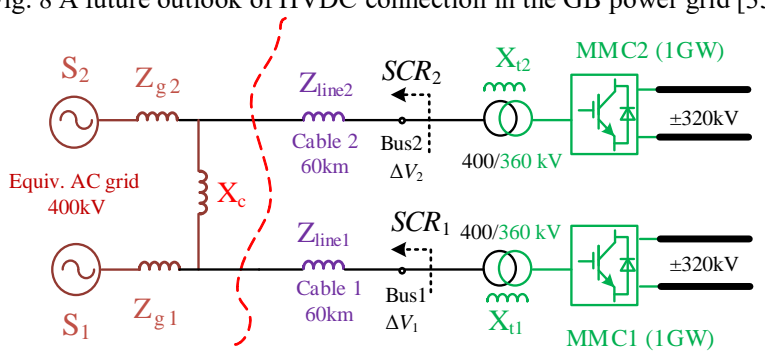

Fig. 9 Equivalent circuit configuration for analytical studies 

is subject to Institution of Electrical and Electronic Engineering Copyright. The copy of record is available at IEEE Xplore Digital Library.

Considering the case with two MMCs, each of the MMC can be equivalent to connection with an $\mathrm{AC}$ source through a certain impedance to emulate the network condition at the $\mathrm{MMC}$ connection point, and the two AC sources are interconnected (within the same AC network). Thus, a simplified network configuration as shown in Fig. 9 can be developed. $Z_{\text {line } 1}$ and $Z_{\text {line2 }}$ in Fig. 9 are considered as the impedances for two $60 \mathrm{~km}$ cables connecting the MMCs to the existing network. The interconnection between the two AC sources is represented by a value of $X_{c}$ considering the high $X / R$ ratio in transmission systems. Applying the MIIF concept, the followings are considered when setting the network parameters:

- $\mathrm{MMC}_{1}$ infeed is considered as an existing HVDC link, and thus $Z_{\mathrm{g} 1}$ is pre-determined.

- When there exists strong electrical coupling between $\mathrm{MMC}_{1}$ and $\mathrm{MMC}_{2}$, i.e. the two converters are in close proximity (high MIIF), $X_{\mathrm{c}}$ is set to a low value while $Z_{\mathrm{g} 2}$ is set to a high value, so that $M \mathrm{MC}_{2}$ can be deemed close to AC system $\mathrm{S}_{1}$ while being further away from $\mathrm{S}_{2}$.

- When there only exists weak electrical coupling between $\mathrm{MMC}_{1}$ and $\mathrm{MMC}_{2}$ (low MIIF), $X_{\mathrm{c}}$ is set to a high value while $Z_{g 2}$ is set to a low value, so that $\mathrm{MMC}_{2}$ can be deemed close to AC system $S_{2}$ and far away from $S_{1}$.

Accordingly, the specific network parameters for cases of weak and strong coupling are given in Table III. The equivalent impedances of the AC grids are $Z_{g 1}=R_{g 1}+j \omega_{0} L_{g 1}$ and $Z_{g 2}=R_{g 2}+j \omega_{0} L_{g 2}$, and $X_{c}=j \omega_{0} L_{c}$ is the reactance for interconnecting the two grids. By varying $Z_{g 1}, Z_{\mathrm{g} 2}$, and $X_{\mathrm{c}}$, different infeed conditions, i.e., electrical distances, can be emulated. Based on the parameters in Table III, the corresponding SCR for weak and strong couplings are presented in Table IV.

Table III System parameter for weak coupling and strong coupling

\begin{tabular}{|c|c|c|}
\hline Parameters & Weak coupling & Strong coupling \\
\hline $\mathrm{L}_{\mathrm{t} 1}$ and $\mathrm{L}_{\mathrm{t} 2}$ & $0.0587 \mathrm{H}$ & $0.0587 \mathrm{H}$ \\
\hline Length of Cable1 \& 2 & $60 \mathrm{~km}$ & $60 \mathrm{~km}$ \\
\hline $\mathrm{R}_{\mathrm{g} 1} / \mathrm{L}_{\mathrm{g} 1}$ & $4.08^{\prime} \Omega / 0.1296 \mathrm{H}$ & $4.08^{\prime} \Omega / 0.1296 \mathrm{H}$ \\
\hline $\mathrm{R}_{\mathrm{g} 2} / \mathrm{L}_{\mathrm{g} 2}$ & $4.08^{\prime} \Omega / 0.1296 \mathrm{H}$ & $10.2 \Omega^{\prime} \Omega / 0.324 \mathrm{H}$ \\
\hline $\mathrm{L}_{\mathrm{c}}$ & $0.3 \mathrm{H}$ & $0.01 \mathrm{H}$ \\
\hline
\end{tabular}

Table IV SCR and MIIF in the case of weak coupling and strong coupling

\begin{tabular}{|c|c|c|}
\hline & Weak coupling & Strong coupling \\
\hline $\mathrm{SCR}_{1} / \mathrm{SCR}_{2}$ & $2.59 / 2.59$ & $2.74 / 2.64$ \\
\hline $\mathrm{MIIF}_{1,2} / \mathrm{MIIF}_{2,1}$ & $0.26 / 0.26$ & $0.78 / 0.81$ \\
\hline
\end{tabular}

Considering the voltages for sources $S_{1}$ and $S_{2}$ are the same, the network can be further simplified by combining the two sources into one with the three delta-connected impedance $Z_{g 1}(s), Z_{g 2}(s)$, and $\mathrm{X}_{\mathrm{c}}(\mathrm{s})$ transformed to equivalent $\mathrm{Y}$ connection as shown in Fig. 10. Note that $\mathbf{Z}_{\mathrm{g} 1}, \mathbf{Z}_{\mathrm{g} 2}$ and $\mathbf{X}_{\mathrm{c}}$ are diagonal 2 by 2 impedance matrix in PN0-frame for the 3-phase balanced system [12].

\section{B. Stability assessment of converters in close proximity}

As shown in Fig. 10, the equivalent AC external impedance $\mathbf{Z}_{\text {eg1 }}$ (s) at $\mathrm{MMC}_{1}$ can be derived as

$$
\begin{aligned}
\mathbf{Z}_{e g 1}(s)= & {\left[\mathbf{Z}_{e 3}(s) \|\left(\mathbf{Z}_{e 2}(s)+\mathbf{Z}_{\text {line } 2}(s)+\mathbf{X}_{t 2}(s)+\mathbf{Z}_{M M C 2}(s)\right]\right.} \\
& +\mathbf{Z}_{e 1}(s)+\mathbf{Z}_{\text {line } 1}(s)+\mathbf{X}_{t 1}(s)
\end{aligned}
$$

According to [33], in order to use Nyquist stability criteria, the system has to meet the following conditions:
- MMC1 and MMC2 are stable when they are individually and directly connected to ideal voltage sources.

- The grid voltage is stable without MMC1 and MMC2 connection.

The above conditions are met in a normal electrical network setup, and the matrices $\mathbf{Z}_{\mathrm{eg} 1}(s)$ and $\mathbf{Y}_{M M C 1}(s)$ do not have righthalf-plane (RHP) poles. Thus, the system stability can be assessed based on Nyquist curve for eigenvalue loci of the matrix $\mathbf{Z}_{\mathrm{eg} 1}(s) \mathbf{Y}_{M M C 1}(s)$. Both $\mathrm{MMC}_{1}$ and $\mathrm{MMC}_{2}$ adopt the same control shown in Fig. 2 and Fig. 3, and also have the same outerloop PQ control with both active references being $1 \mathrm{GW}$. The reactive power of the MMCs are set to maintain their terminal voltages at $400 \mathrm{kV}$, and the control parameters are listed in Table V.

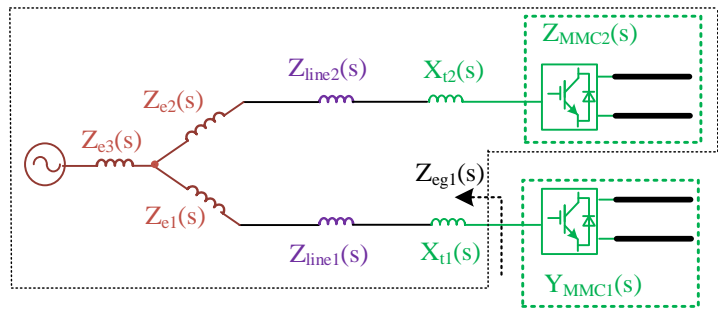

Fig. 10 Small-signal impedance equivalent circuit

Table V Controller parameters for MMC controller

\begin{tabular}{|c|c|}
\hline Parameters & Value \\
\hline Current loop PI gains: $K_{i p}, K_{i i}$ & $15.8 \Omega, 2980 \Omega / \mathrm{s}$ \\
\hline PLL PI gains: $K_{p l l p}, K_{p l l i}$ & $0.0013 \mathrm{rad} /(\mathrm{sV}), 0.12 \mathrm{rad} /\left(\mathrm{s}^{2} \mathrm{~V}\right)$ \\
\hline CCSC PR controller gains: $K_{r p}, K_{r r}$ & $63.3 \Omega, 11200 \Omega / \mathrm{s}$ \\
\hline AC voltage controller PI gains: $K_{r p}, K_{r i}$ & $0.005 \mathrm{~A} / \mathrm{V}, 0.5 \mathrm{~A} /(\mathrm{s} \cdot \mathrm{V})$ \\
\hline
\end{tabular}

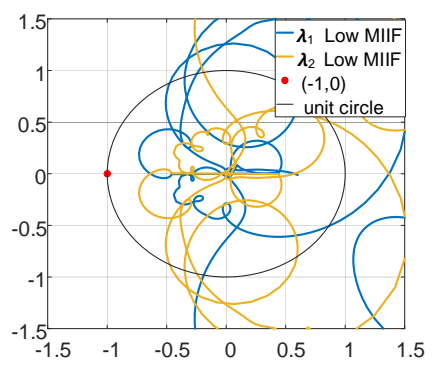

(a) with low MIIF

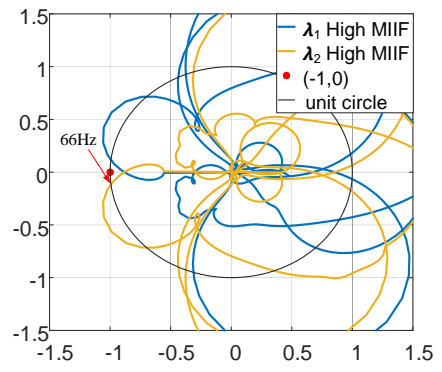

(b) with high MIIF
Fig. 11 Nyquist plots for different MIIF using PQ control

Under different MIIF, the Nyquist plots for eigenvalue loci of $\mathbf{Z}_{\mathrm{eg} 1}(s) \mathbf{Y}_{M M C 1}(s)$ are compared in Fig. 11. For both MIIF cases, the eigenvalue locus do not encircle the point $(-1,0)$ and thus the system is stable. As described in [37], the phase and gain margins can also be observed based on the Nyquist plots. With low MIIF ( $\mathrm{MIIF}_{2,1}=0.26$ in this example), the interaction of the two MMCs are weak and the Nyquist plots imply that the system has sufficient phase margin and magnitude margin and thus system stability is strong. In the case of high MIIF $\left(\mathrm{MIIF}_{2,1}=0.81\right.$ in this example), the system stability is weakened with low gain margin and phase margin. Meanwhile, the crossover frequency of the Nyquist curve shown in Fig. 11(b) is $66 \mathrm{~Hz}$, indicating the system has the worst stability around $66 \mathrm{~Hz}$, which is in the frequency range of MMC negative resistance appearing in Fig. 7. However, it needs to be noted that MMC negative resistance itself will not necessarily lead to unstable system as stability is the result of the interaction of multiple impedances including network. 
The corresponding time-domain simulation results are given in Fig. 12. At 12s, a small perturbation is injected into the active power reference of MMC1. The d-axis current of MMC1 with low MIIF has smaller overshoot and can reach stable operation quicker than that under high MIIF as seen in Fig. 12 (a) and (b), indicating the system under low MIIF has higher stability margin than that under high MIIF. Note that the oscillation frequency in Fig. 12 (b) is around $16 \mathrm{~Hz}$ in dq-frame, corresponding to $66 \mathrm{~Hz}$ in $\mathrm{AC}$ system. The simulation results thus accord well with the Nyquist analysis.

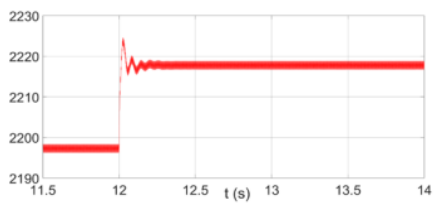

(a) Low MIIF

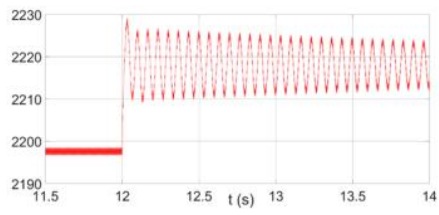

(b) High MIIF
Fig. 12 The d-axis current of MMC1 with different MIIF

The effect of different outer-loop control on the stability of the interconnection system is further investigated. MMC1 now adopts PQ control and MMC2 PV control. Under different MIIF, the Nyquist plots are depicted in Fig. 13. As can be seen, with low MIIF, the system can maintain sufficient stability, whereas with high MIIF, the system becomes unstable. The corresponding time-domain simulation results shown in Fig. 14 match well with that of Fig. 13, in which the system is unstable for high MIIF. Further studies considering different MIIF and outer-loop controllers reveal similar results. When multiple converters are considered, a system with low MIIF has better system stability than that with high MIIF, and outer-loop can also significantly impact on system stability. However, a clear boundary between high and low MIIF is difficult to define and full system studies are required to determine system stability.

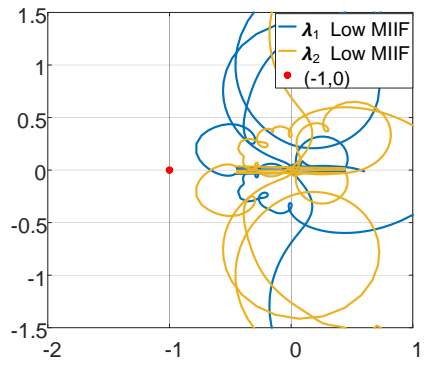

(a) Low MIIF

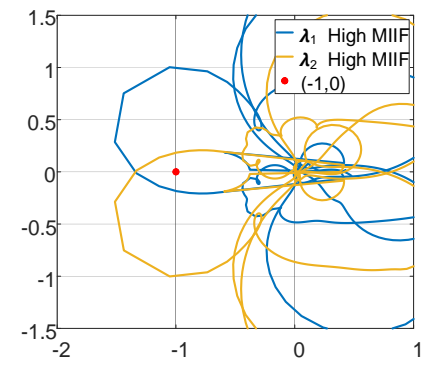

(b) High MIIF

Fig. 13 Nyquist plots with PQ and PV control.

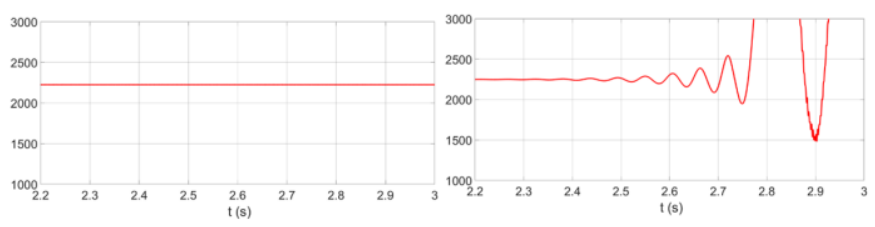

(a) Low MIIF

(b) High MIIF

Fig. 14 The d-axis current of MMC1 with different MIIF.

Looking into the causes of the reduced stability margin in Fig. 11(b) and instability in Fig. 13(b), the negative resistance in the MMC admittance in the frequency range of $50-70 \mathrm{~Hz}$ is of clear concern. This in combination with the high admittance magnitude under PV control leads to instability as shown in Fig. 13(b) and Fig. 14(b). Therefore, parameter turning or additional voltage control to reduce the admittance magnitude within 50-
$70 \mathrm{~Hz}$ under PV control can likely improve system stability. Further studies and results will be report in the future.

When assessing system stability, converters including wind farms, HVDC, FACTS etc. connected in close proximity to the point of common coupling must be fully considered. In addition, converter admittance is affected by its operating point as previously described. Thus, the assessment of multiple converter interaction is a complex issue which is affected by multiple factors including the states and operation of the network and converters etc.

\section{CONCLUSION}

This paper has described the impedance modelling and validation of the three-phase MMC converter based on HSS. The detailed mathematical expressions for HSS modelling for MMC have been derived considering the integration of various inner and outer control loops. The coupling between the positive and negative sequence components brought by external control loops and PLL are analyzed in the model. The smallsignal impedances obtained from the developed analytical model have been validated using time-domain models. With the impedance model, the interaction of multiple converters in close proximity is studied considering different multi-infeed interaction factor (MIIF). Stability analysis and time domain simulation results show good match and that system with high MIIF where strong couplings between the two MMCs exist may cause the instability of the system.

Converter and system impedances are highly dependent on operating point, controller setting, and network structure, and thus further studies to investigate the impact of their variations on system stability are required. In addition, to identify states where the risk of instability may exist in a multi-infeed converter system is critical, so as to help inform operating away from those network or converter operating states.

\section{APPENDIX A}

The matrices in (14) are given as

$$
\begin{aligned}
\Gamma\left[\mathbf{A}_{s}\right] & =\left[\begin{array}{cccccccc}
\mathbf{A}_{s}^{0} & \mathbf{A}_{s}^{-1} & \ldots & \mathbf{A}_{s}^{-h} & & & \\
\mathbf{A}_{s}^{1} & \ddots & \ddots & \ddots & \ddots & & \\
\vdots & \ddots & \mathbf{A}_{s}^{0} & \mathbf{A}_{s}^{-1} & \ddots & \ddots & \\
\mathbf{A}_{s}^{h} & \ldots & \mathbf{A}_{s}^{1} & \mathbf{A}_{s}^{0} & \mathbf{A}_{s}^{-1} & \ldots & \mathbf{A}_{s}^{-h} \\
& \ddots & \ddots & \mathbf{A}_{s}^{1} & \mathbf{A}_{s}^{0} & \ddots & \vdots \\
& & \ddots & \ddots & \ddots & \ddots & \mathbf{A}_{s}^{-1} \\
& & & \mathbf{A}_{s}^{h} & \ldots & \mathbf{A}_{s}^{1} & \mathbf{A}_{s}^{0}
\end{array}\right] \\
\Gamma[\mathbf{B}] & =\left[\begin{array}{cccccccc}
\mathbf{B}^{0} & \mathbf{B}^{-1} & \ldots & \mathbf{B}^{-h} & & & \\
\mathbf{B}^{1} & \ddots & \ddots & \ddots & \ddots & & \\
\vdots & \ddots & \mathbf{B}^{0} & \mathbf{B}^{-1} & \ddots & \ddots & \\
\mathbf{B}^{h} & \ldots & \mathbf{B}^{1} & \mathbf{B}^{0} & \mathbf{B}^{-1} & \ldots & \mathbf{B}^{-h} \\
& \ddots & \ddots & \mathbf{B}^{1} & \mathbf{B}^{0} & \ddots & \vdots \\
& & \ddots & \ddots & \ddots & \ddots & \mathbf{B}^{-1} \\
& & & \mathbf{B}^{h} & \ldots & \mathbf{B}^{1} & \mathbf{B}^{0}
\end{array}\right]
\end{aligned}
$$


This paper is a post-print of a paper submitted to and accepted for publication in IEEE Journal of Emerging and Selected Topics in Power Electronics and is subject to Institution of Electrical and Electronic Engineering Copyright. The copy of record is available at IEEE Xplore Digital Library.

$$
\Gamma[\mathbf{M}]=\left[\begin{array}{ccccccc}
\mathbf{M}^{0} & \mathbf{M}^{-1} & \ldots & \mathbf{M}^{-h} & & & \\
\mathbf{M}^{1} & \ddots & \ddots & \ddots & \ddots & & \\
\vdots & \ddots & \mathbf{M}^{0} & \mathbf{M}^{-1} & \ddots & \ddots & \\
\mathbf{M}^{h} & \ldots & \mathbf{M}^{1} & \mathbf{M}^{0} & \mathbf{M}^{-1} & \ldots & \mathbf{M}^{-h} \\
& \ddots & \ddots & \mathbf{M}^{1} & \mathbf{M}^{0} & \ddots & \vdots \\
& & \ddots & \ddots & \ddots & \ddots & \mathbf{M}^{-1} \\
& & & \mathbf{M}^{h} & \ldots & \mathbf{M}^{1} & \mathbf{M}^{0}
\end{array}\right]
$$

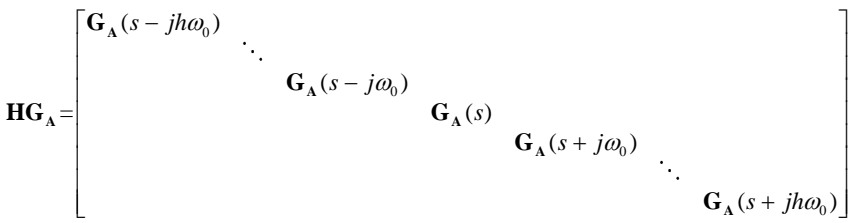

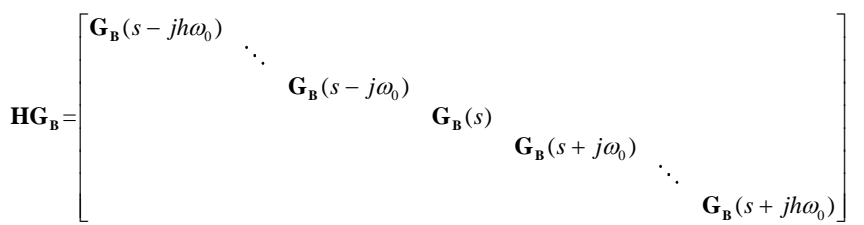<smiles>CC(C)C1(O)CCCC1</smiles>

$$
\Delta \mathbf{X}_{P N 0}=\left[\begin{array}{c}
\Delta \mathbf{x}_{P N 0}\left(s-j h \omega_{0}\right) \\
\vdots \\
\Delta \mathbf{x}_{P N 0}\left(s-j \omega_{0}\right) \\
\Delta \mathbf{x}_{P N 0}(s) \\
\Delta \mathbf{x}_{P N 0}\left(s+j \omega_{0}\right) \\
\vdots \\
\Delta \mathbf{x}_{P N 0}\left(s+j h \omega_{0}\right)
\end{array}\right], \quad \Delta \mathbf{V}_{P N 0}=\left[\begin{array}{c}
\Delta \mathbf{v}_{P N 0}\left(s-j h \omega_{0}\right) \\
\vdots \\
\Delta \mathbf{v}_{P N 0}\left(s-j \omega_{0}\right) \\
\Delta \mathbf{v}_{P N 0}(s) \\
\Delta \mathbf{v}_{P N 0}\left(s+j \omega_{0}\right) \\
\vdots \\
\Delta \mathbf{v}_{P N 0}\left(s+j h \omega_{0}\right)
\end{array}\right]
$$

\section{REFERENCES}

[1] H. Saad, Y. Fillion, S. Deschanvres, Y. Vernay, and S. Dennetière, "On resonances and harmonics in HVDC-MMC station connected to AC grid," IEEE Trans. Power Del., vol. 32, no. 3, pp. 1565-1573, Jun. 2017.

[2] A. Beddard, C. E. Sheridan, M. Barnes, and T. C. Green, "Improved accuracy average value models of modular multilevel converters," IEEE Trans. Power Del., vol. 31, no. 5, pp. 2260-2269, Oct. 2016.

[3] H. Yang, Y. Dong, W. Li, and X. He, "Average-value model of modular multilevel converters considering capacitor voltage ripple," IEEE Trans. Power Del., vol. 32, no. 2, pp. 723-732, Apr. 2017.

[4] J. Dorn, H. Huang, and D. Retzmann, "A new multilevel voltage-sourced converter topology for HVDC applications," in Proc. CIGRE, Paris, France, Aug. 24-29, 2008, pp. 1-8.

[5] S. Shah, "Small and large signal impedance modelling for stability analysis of grid-connected voltage source converters," Department of Electrical Engineering, Rensselaer Polytechnic Institute, Troy, New York, 2018.

[6] A. Nami, J. Liang, F. Dijkhuizen, and G. D. Demetriades, "Modular multilevel converters for HVDC applications: Review on converter cells and functionalities," IEEE Trans. Power Electron., vol. 30, no. 1, pp. 18 36, Jan. 2015.

[7] A. Jamshidifar, and D. Jovcic, "Small-signal dynamic dq model of modular multilevel converter for system studies," IEEE Trans. Power Del., vol. 31, no. 1, pp. 1991-1999, Feb. 2016.

[8] Y. Li, G. Tang, J. Ge, Z. He, H. Pang, J. Yang, and Y. Wu, "Modelling and damping control of modular multilevel converter based dc grid," IEEE Trans. Power Syst., vol. 31, no. 1, pp. 723-735, Jan. 2018.
[9] J. Lyu, X. Cai, and M. Molinas, "Frequency domain stability analysis of MMC-based HVDC for wind farm integration," IEEE J. Emerg. Sel.Topics Power Electron., vol. 4, no. 1, pp. 141-151, Mar. 2016.

[10] M. Beza, M. Bongiorno, and G. Stamatiou, "Analytical derivation of the ac-side input admittance of a modular multilevel converter with open- and closed-loop control strategies," IEEE Trans. Power Del., vol. 33, no. 1, pp. 248-256, Feb. 2018.

[11] L. Bessegato, K. Ilves, L. Harnefors, and S. Norrga, "Effects of control on the ac-side admittance of a modular multilevel converter", IEEE Trans. Power Electron., vol. 34, no. 8, pp. 7206-7220, Aug. 2019.

[12] S. Hwang, "Harmonic state-space modelling of an hvdc converter with closed-loop control," Department of Electrical and Computer Engineering, University of Canterbury, Christchurch, New Zealand, 2013.

[13] J. J. Rico, M. Madrigal and E. Acha, "Dynamic harmonic evolution using the extended harmonic domain," IEEE Trans. Power Del., vol. 18, no. 2, pp. 587-594, April 2003.

[14] J. J. Chavez and A. Ramirez, "Dynamic Harmonic Domain Modelling of Transients in Three-Phase Transmission Lines," IEEE Trans. Power Del., vol. 23, no. 4, pp. 2294-2301, Oct. 2008.

[15] J. B. Kwon, X. Wang, F. Blaabjerg, C. L. Bak, A. R. Wood and N. R. Watson, "Harmonic instability analysis of a single-phase grid-connected converter using a harmonic state-space modelling method," IEEE Trans. Ind. Appl., vol. 52, no. 5, pp. 4188-4200, Sept.-Oct. 2016.

[16] J. Lyu, X. Zhang, X. Cai, and M. Molinas, "Harmonic state-space based small-signal impedance modelling of modular multilevel converter with consideration of internal harmonic dynamics," IEEE Trans. Power Electron., vol. 34, no. 3, pp. 2134-2148, Mar. 2019.

[17] Z. Xu, B. Li, S. Wang, S. Zhang and D. Xu, "Generalised Single-Phase Harmonic State Space Modelling of the Modular Multilevel Converter With Zero-Sequence Voltage Compensation," IEEE Trans. Ind. Electron., vol. 66, no. 8, pp. 6416-6426, Aug. 2019.

[18] H. Wu, X. Wang and Ł. Kocewiak, "Impedance-Based Stability Analysis of Voltage-Controlled MMCs Feeding Linear AC Systems," IEEE J. Emerg. Sel. Topics Power Electron., early access, 2019.

[19] H. Wu and X. Wang, "Dynamic Impact of Zero-Sequence Circulating Current on Modular Multilevel Converters: Complex-Valued AC Impedance Modelling and Analysis," IEEE J. Emerg. Sel. Topics Power Electron., vol. 8, no. 2, pp. 1947-1963, June 2020.

[20] A. Rygg, M. Molinas, C. Zhang and X. Cai, "A Modified SequenceDomain Impedance Definition and Its Equivalence to the dq-Domain Impedance Definition for the Stability Analysis of AC Power Electronic Systems," IEEE J. Emerg. Sel. Topics Power Electron., vol. 4, no. 4, pp. 1383-1396, Dec. 2016.

[21] H. Zong, J. Lyu, C. Zhang, X. Cai, M. Molinas and F. Rao, "MIMO impedance based stability analysis of DFIG-based wind farm with MMCHVDC in modified sequence domain," in Proc. 8th Int. Conf. Renew. Power Gener. (RPG), Shanghai, China, 2019, pp. 1-7.

[22] H. Zong, C. Zhang, J. Lyu, X. Cai, M. Molinas and F. Rao, "Generalized MIMO Sequence Impedance Modeling and Stability Analysis of MMCHVDC With Wind Farm Considering Frequency Couplings," IEEE Access, vol. 8, pp. 55602-55618, 2020.

[23] K. Ji, G. Tang, H. Pang and J. Yang, "Impedance Modeling and Analysis of MMC-HVDC for Offshore Wind Farm Integration," IEEE Trans. Power Del., vol. 35, no. 3, pp. 1488-1501, June 2020

[24] R. Li, L. Xu and D. Guo, "Accelerated switching function model of hybrid MMCs for HVDC system simulation," IET Power Electron., vol. 10, no. 15, pp. 2199-2207, 15122017.

[25] D. Guo, M. H. Rahman, G. P. Ased, L. Xu, A. Emhemed, G. Burt, et al., "Detailed quantitative comparison of half-bridge modular multilevel converter modelling methods," The Journal of Engineering, vol. 2019, pp. 1292-1298, 2019

[26] J. Sun, "Small-Signal Methods for AC Distributed Power Systems-A Review," IEEE Trans. Power Electron., vol. 24, no. 11, pp. 2545-2554, Nov. 2009.

[27] D. N. Zmood and D. G. Holmes, "Stationary frame current regulation of PWM inverters with zero steady-state error," IEEE Trans. Power Electron., vol. 18, no. 3, pp. 814-822, May. 2003.

[28] B. Wen, D. Boroyevich, R. Burgos, P. Mattavelli, and Z. Shen, "Analysis of D-Q small-signal impedance of grid-tied inverters," IEEE Trans. Power Electron., vol. 31, no. 1, pp. 675-687, Jan. 2016.

[29] G. Amico, A. Egea-Àlvarez, P. Brogan and S. Zhang, "Small-signa converter admittance in the pn-frame: systematic derivation and analysis of the cross-coupling terms," IEEE Trans. Energy Conver., vol. 34, no. 4, pp. $1829-1838$, Dec. 2019. 
This paper is a post-print of a paper submitted to and accepted for publication in IEEE Journal of Emerging and Selected Topics in Power Electronics and is subject to Institution of Electrical and Electronic Engineering Copyright. The copy of record is available at IEEE Xplore Digital Library.

[30] O. Katsuhiko and Y. Yang, Modern Control Engineering, 5ed. Upper Saddle River, NJ: Prentice hall, 2010.

[31] J. Sun and H. Liu, "Sequence Impedance Modelling of Modular Multilevel Converters," IEEE J. Emerg. Sel. Topics Power Electron., vol. 5, no. 4, pp. 1427-1443, Dec. 2017.

[32] M. Cespedes and J. Sun, "Impedance Modelling and Analysis of GridConnected Voltage-Source Converters," IEEE Trans. Power Electron., vol. 29, no. 3, pp. 1254-1261, March. 2014.

[33] J. Sun, "Impedance-based stability criterion for grid-connected inverters," IEEE Trans. Power Electron., vol. 26, no. 11, pp. 3075-3078, Nov. 2011.

[34] M. Cespedes, L. Xing and J. Sun, "Constant-power load system stabilisation by passive damping," IEEE Trans. Power Electron., vol. 26, no. 7, pp. 1832-1836, July 2011.

[35] "GB National Electricity System Seven Year Statement", National Grid, 2011.

[36] B. Davies et al., Systems with multiple dc infeed, CIGRE Working Group B4.41, Paris, France, pp. 12-14, Dec. 2008.

[37] L. Harnefors, "Modeling of Three-Phase Dynamic Systems Using Complex Transfer Functions and Transfer Matrices," IEEE Trans. Ind. Electron., vol. 54, no. 4, pp. 2239-2248, Aug. 2007.

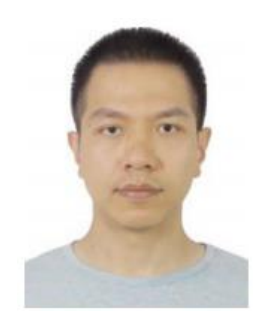

Yin Chen received the B.S. degree in electrical engineering from Huazhong University of Science and Technology, Wuhan, China, in 2009, and the M.S. degree in electrical engineering from Zhejiang University, Hangzhou, China, in 2014. He received the $\mathrm{Ph} . \mathrm{D}$. degree in Electrical Engineering from University of Strathclyde, Glasgow, U.K. in 2020.

$\mathrm{He}$ is currently a post-doctoral researcher with University of Strathclyde in Glasgow, UK. His research interests include modelling of power electronic converters, grid integration of renewable power, and stability analysis of the HVDC transmision systems.

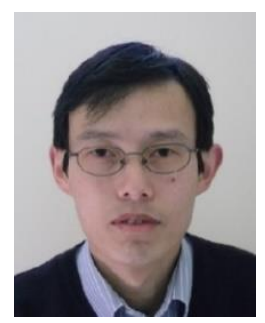

Lie Xu (M'03-SM'06) received the B.Sc. degree in Mechatronics from Zhejiang University, Hangzhou, China, in 1993, and the Ph.D. degree in Electrical Engineering from the University of Sheffield, Sheffield, UK, in 2000.

$\mathrm{He}$ is currently a Professor at the Department of Electronic \& Electrical Engineering, University of Strathclyde, Glasgow, UK. He previously worked in Queen's University of Belfast and ALSTOM T\&D, Stafford, UK. His current research interests include power electronics, wind energy generation and grid integration, and application of power electronics to power systems such as HVDC and MVDC systems for power transmission and distribution. He is an Editor of IEEE Transactions on Power Delivery and IEEE Transactions on Energy Conversion.

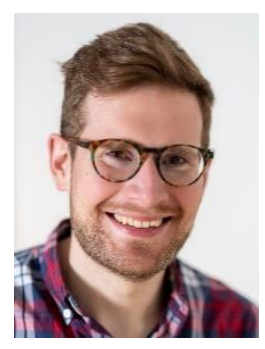

Agustí Egea-Àlvarez (S'12-M'14) obtained his B.Sc, MSc and Ph.D. from the Technical University of Catalonia in Barcelona in 2008, 2010 and 2014 respectively. In 2015 he was a Marie Curie fellow in the China Electric Power Research Institute (CEPRI). In 2016 he joined Siemens Gamesa as converter control engineer working on grid forming controllers and alternative HVDC schemes for offshore wind farms. Currently, Dr Agust Egea-lvarezis Strathclyde Chancellors fellow (Lecturer) at the electronic \& electrical engineering department and member of the PEDEC (Power Electronics, Drives and Energy Conversion) group since 2018. $\mathrm{He}$ is a member of IEEE, IET and has been involved in several CIGRE and ENTSO-E working groups.

His current research interests include control and operation of high-voltage direct current systems, renewable generation systems, electrical machines and power converter control.

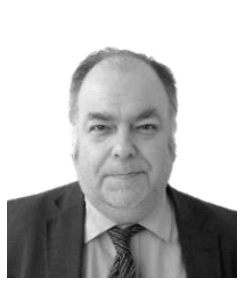

Benjamin Marshall is currently the HVDC Technology Manager at the UK National HVDC Centre. Ben oversees the team of Simulation Engineers undertaking detailed HVDC simulation studies in realtime using vendor-supplied replica hardware, to understand multi-infeed, multi-terminal and multivendor HVDC operation and interactions, for real schemes in GB; interpreting the results to gain insights to improve the design and operation of HVDC schemes.

Ben previously has had a 23 year long and varied career within National Grid with a broad range of experience, particularly with respect to the analysis of the operation and design of the AC and DC transmission systems. He has experience in both offline and real-time EMT simulation and in modelling of convertors across battery, solar wind and HVDC systems, He has developed deep technical skills relating to dynamic stability of power systems and the performance specification of HVDC convertors. Within the ESO, Ben advised on the specification, validation and modelling of new HVDC connections, supporting the compliance connection planning and requirements and provided technical leadership on AC and DC control systems, System Operability, Smart Grids and power system simulation; leading complex power system studies.

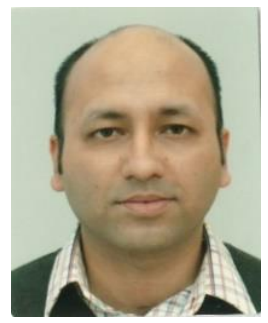

Md Habibur Rahman obtained B.Sc. degree in Electrical and Electronic Engineering from Ahsanullah University of Science and Technology, Bangladesh in 2007, and in 2011 he received his M.Sc. degree in Sustainable Electrical Power from Brunel University, UK. Since 2014 has been a PhD researcher with the Department of Electronic and Electrical Engineering at the University of Strathclyde, UK.

$\mathrm{He}$ is currently a simulation engineer at the National HVDC Centre, UK. His research interest is mainly focused on Multiterminal HVDC system concentrating on fault management, design of converters for HVDC applications, protection schemes and control of power electronics. He is an active contributor to the reviewing process of IEEE and IET Transactions and conferences and a member of IEEE and IET.

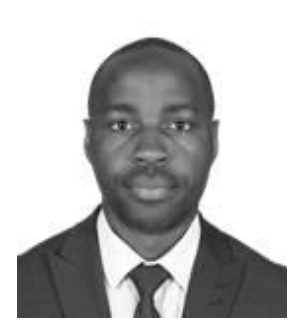

Oluwole Daniel Adeuyi (S'11-M'16) received the B.Sc. degree in electrical/electronic engineering from the Federal University of Agriculture, Abeokuta, Nigeria, in 2010, the M.Sc. degree (Hons.) in 2012, and the Ph.D. degree in electrical energy system in 2016, both from Cardiff University, U.K, where he worked as a postdoctoral research associate with focus on control and operation of HVDC-connected wind farms. In 2018, he joined the National HVDC Centre (part of SSEN Transmission) and has supported de-risking industrial HVDC schemes for integration of renewable energy systems. 